\title{
Thermodynamics of the Classical Planar Ferromagnet Close to the Zero-Temperature Critical Point: A Many-Body Approach
}

\author{
L. S. Campana, ${ }^{1}$ A. Cavallo, ${ }^{2}$ L. De Cesare, ${ }^{2,3}$ U. Esposito, ${ }^{1}$ and A. Naddeo $^{2,3}$ \\ ${ }^{1}$ Dipartimento di Scienze Fisiche, Università degli Studi di Napoli “Federico II", Piazzale Tecchio 80, 80125 Napoli, Italy \\ ${ }^{2}$ Dipartimento di Fisica "E.R. Caianiello", Università degli Studi di Salerno, Via Ponte don Melillo, 84084 Fisciano, Italy \\ ${ }^{3}$ CNISM, Unità di Ricerca di Salerno, 84084 Fisciano, Italy
}

Correspondence should be addressed to A. Cavallo, cavallo@sa.infn.it

Received 1 February 2012; Accepted 15 March 2012

Academic Editor: Giancarlo Consolo

Copyright ( 2012 L. S. Campana et al. This is an open access article distributed under the Creative Commons Attribution License, which permits unrestricted use, distribution, and reproduction in any medium, provided the original work is properly cited.

\begin{abstract}
We explore the low-temperature thermodynamic properties and crossovers of a $d$-dimensional classical planar Heisenberg ferromagnet in a longitudinal magnetic field close to its field-induced zero-temperature critical point by employing the two-time Green's function formalism in classical statistical mechanics. By means of a classical Callen-like method for the magnetization and the Tyablikov-like decoupling procedure, we obtain, for any $d$, a low-temperature critical scenario which is quite similar to the one found for the quantum counterpart. Remarkably, for $d>2$ the discrimination between the two cases is found to be related to the different values of the shift exponent which governs the behavior of the critical line in the vicinity of the zero-temperature critical point. The observation of different values of the shift-exponent and of the related critical exponents along thermodynamic paths within the typical V-shaped region in the phase diagram may be interpreted as a signature of emerging quantum critical fluctuations.
\end{abstract}

\section{Introduction}

An intriguing aspect of quantum phase transitions (QPTs) [1] is that quantum critical fluctuations may play a relevant role also at finite temperature. This feature leads to a drastic modification of the expected properties of many systems within a wide region around their quantum critical point (QCP) [1-6].

Remarkably, the renormalization group framework (RG) $[1,7,8]$ and Moriya's self-consistent renormalized approach $[9,10]$ have provided a well-defined scenario for this socalled quantum criticality giving qualitative and also quantitative agreement with a lot of experimental findings [1-6].

However, recent experiments seem to suggest that these theories fail in relevant practical situations (see [11-13] and references therein). Although several alternatives have been proposed to explain these unexpected behaviors, a completely convincing picture is still lacking. Hence, it becomes crucial to provide nonambiguous criteria to determine accurately the range of temperatures where the QCP fluctuations survive against the thermal ones. On the ground of a comparison between the exactly solvable one-dimensional quantum transverse Ising model (QTIM) $[1,14-18]$ and its classical version (CTIM) (not to be confused with the standard Ising model) $[19,20]$, it was conjectured that [21], at least in selected cases, at finite temperature, close to the QCP, quantum critical fluctuations may not be so relevant as commonly believed. The emerging idea was that, to single out conventional quantum criticality, it is not sufficient to observe a power-law behavior of the correlation length or susceptibility decreasing temperature towards zero in the Vshaped quantum critical region of the phase diagram [1]; rather, the accurate determination of the critical exponents becomes the key ingredient to decide if we are in the influence domain of the QCP or the physics is governed by thermal fluctuations. Of course, to validate the previous 
conjecture, more realistic many-body systems should be investigated, especially for dimensionalities where a finitetemperature critical line ends in a zero-temperature critical point.

A first step along this direction has been recently performed by exploring the low-temperature properties of the $(d>1)$-dimensional CTIM [22]. On the ground of a suitable Ginzburg-Landau-Wilson functional and a momentumshell RG approach around $d=4$, this system is expected to have the same properties of the QTIM above a certain temperature.

Further insights will be provided in this paper where we investigate the low-temperature properties of the classical XXZ ferromagnetic model in presence of a longitudinal magnetic field when the longitudinal exchange interaction is smaller than the transverse one. Its quantum analogue, also called planar ferromagnet (PFM), has been extensively studied, in different physical contexts, using several methods. In particular, the spin-1/2 PFM has attracted great attention since, in the pioneering papers by Matsubara and Matsuda $[23,24]$ on superfluidity in ${ }^{4} \mathrm{He}$, a quantum lattice gas of hard-core bosons with long-range attractive interactions has been proven to be just equivalent to the spin-1/2 PFM in a longitudinal field (see also [25]). Remarkably, the Wilson RG $[26,27]$, applied to a suitable functional representation of the spin-1/2 PFM, capturing the essential low-temperature physics, and the two-time Green's function technique [28], utilized to investigate the microscopic spin- $S$ model, have provided a reliable scenario of the global phase diagram and crossovers in the vicinity of the QCP.

In the present work, we will study the corresponding $d$ dimensional classical XXZ spin model (CPFM) with particular attention to a field-induced quantum-like critical scenario for a direct comparison with the quantum counterpart. We will use the two-time Green's function method in classical statistical mechanics [29], developed and tested in [3033 ], on microscopic classical spin model. This allows us to perform in parallel the quantum [28] and the classical analysis for any $d$, giving a transparent relation between the CPFM and the spin-S QPFM, both exhibiting a zerotemperature critical point. Hence, new insights on quantum criticality, at least for a class of anisotropic magnetic systems, will be provided.

The paper is organized as follows. In Section 2 we will introduce the model and the equation of motion for the appropriate two-time Green function in the context of the classical Callen-like method [34] to calculate the magnetization within the Tyablikov decoupling procedure. The equations for the transverse susceptibility and the critical line will be presented in Section 3. The quantum-like scenario, with the global phase diagram and crossovers, close to the $(T=0)$-critical point, will be analyzed in Section 4. In Section 5, concluding remarks will be drawn. At the end, for utility of reader, Appendix A is devoted to an outline of the two-time Green's function framework in classical statistical mechanics and Appendix B presents a method, alternative to the one employed in [22], to obtain the magnetization as the solution of the Callen-like method.

\section{Spin Model and Callen-Like Method}

The $d$-dimensional classical XXZ Heisenberg model in a longitudinal magnetic field $h \geq 0$ is described by the Hamiltonian:

$$
\begin{aligned}
\mathscr{H} & =-\frac{1}{2} \sum_{i, j=1}^{N}\left[J_{i j}^{\perp}\left(S_{i}^{x} S_{j}^{x}+S_{i}^{y} S_{j}^{y}\right)+J_{i j}^{\|} S_{i}^{z} S_{j}^{z}\right]-h \sum_{i=1}^{N} S_{i}^{z} \\
& =-\frac{1}{2} \sum_{i, j=1}^{N}\left[J_{i j}^{\perp} S_{i}^{+} S_{j}^{-}+J_{i j}^{\|} S_{i}^{z} S_{j}^{z}\right]-h \sum_{i=1}^{N} S_{i}^{z} .
\end{aligned}
$$

Here $\left\{\mathbf{S}_{j} \equiv\left(S_{j}^{x}, S_{j}^{y}, S_{j}^{z}\right) ; j=1,2, \ldots, N\right\}$ are classical spin- $S$ vectors on an $N$-sites hypercubic lattice with unitary spacing, satisfying the identity $S_{j}^{2}=S_{j}^{+} S_{j}^{-}+\left(S_{j}^{z}\right)^{2}=S^{2}$ with $S_{j}^{ \pm}=S_{j}^{x} \pm$ $i S_{j}^{y}$. Besides, the transverse $\left(J_{i j}^{\perp}\right)$ and longitudinal $\left(J_{i j}^{\|}\right)$exchange interactions (with $J_{i i}^{\perp}=J_{i i}^{\|}=0$ ) between the spins at sites $i$ and $j$ are assumed to be symmetric, positive, and short ranged. As well known, it is perfectly legal in the classical context to put $S=1$. However, for a more transparent and direct comparison with the quantum version [28], through this paper we will consider arbitrary $S$.

Many magnetic materials can be described by the Hamiltonian (1) and different cases may take place depending on the relative weight of the competing anisotropic exchange interactions. Indeed, one has a uniaxial ferromagnet (FM) if $J_{i j}^{\|}>J_{i j}^{\perp}$ with the extreme limit $J_{i j}^{\perp}=0$ (Ising model); we recover the isotropic Heisenberg model when $J_{i j}^{\perp}=J_{i j}^{\|}$and the PFM if $J_{i j}^{\|}<J_{i j}^{\perp}$ whose extreme limit $J_{i j}^{\|}=0$ is the XY model in a transverse field (TXYM). In the following, we will focus on the classical PFM which exhibits a field-driven zerotemperature critical point as it happens in the quantum case.

The classical model (1) can be described in terms of the $2 N$ canonical variables $\phi \equiv\left\{\phi_{j}\right\}$ and $S^{z} \equiv\left\{S_{j}^{z}\right\}$, where $\phi_{j}$ is the angle between the projection of the spin vector $S_{j}$ in the $x y$-plane and the $x$-axis. The Poisson bracket of two generic classical dynamical variables $A=A\left(\phi, S^{z}\right)$ and $B=B\left(\phi, S^{z}\right)$ is then defined by

$$
\{A, B\}=\sum_{j=1}^{N}\left(\frac{\partial A}{\partial \phi_{j}} \frac{\partial B}{\partial S_{j}^{z}}-\frac{\partial A}{\partial S_{j}^{z}} \frac{\partial B}{\partial \phi_{j}}\right) .
$$

It is easy to show that, with this prescription, the Poisson brackets for the spin components are given by

$$
\begin{gathered}
\left\{S_{i}^{z}, S_{j}^{ \pm}\right\}=\mp i S_{i}^{ \pm} \delta_{i j}, \\
\left\{S_{i}^{+}, S_{j}^{-}\right\}=-2 i S_{i}^{z} \delta_{i j}, \\
\left\{S_{i}^{\alpha}, S_{j}^{\beta}\right\}=\epsilon_{\gamma}^{\alpha \beta} S_{i}^{\gamma} \delta_{i j} \quad(\alpha, \beta, \gamma=x, y, z),
\end{gathered}
$$

where $\epsilon_{\gamma}^{\alpha \beta}$ is the Levi-Civita tensor. 
Following the Callen procedure developed for the quantum Heisenberg FM [35], we introduce now the retarded two-time GF $[29,33]$ (see Appendix A):

$$
\begin{aligned}
G_{i j}\left(t-t^{\prime}\right) & =\theta\left(t-t^{\prime}\right)\left\langle\left\{S_{i}^{+}\left(t-t^{\prime}\right), e^{a S_{j}^{z}} S_{j}^{-}\right\}\right\rangle \\
& =\left\langle\left\langle S_{i}^{+}\left(t-t^{\prime}\right) ; e^{a S_{j}^{z}} S_{j}^{-}\right\rangle\right\rangle,
\end{aligned}
$$

where $\theta(x)$ is the usual step function, $a$ denotes the Callenlike parameter, $\langle\cdots\rangle=\mathcal{Z}^{-1} \prod_{j=1}^{N} \int_{0}^{2 \pi} d \phi_{j} \int_{-S}^{S} \cdots d S_{j}^{z} \cdots$ $\exp \left\{-\beta \mathcal{H}\left(\phi, S^{z}\right)\right\}$ stands for the classical ensemble average, $\beta=1 / T$ is the inverse temperature, and $X(t)=e^{i L t} X, L=$ $i\{\mathscr{H}, \ldots\}$ is the Liouville operator. Here, $e^{i L t}$ acts as a classical time-evolution operator which transforms the dynamical variable $X=X(0) \equiv X\left(\phi(0), S^{z}(0)\right)$ at the initial time $t=0$ into $X(t) \equiv X\left(\phi(t), S^{z}(t)\right)$ at the time $t$. The physics will be of course obtained setting $a=0$ at the end of the calculations.

The equation of motion (EM) for the GF (4) is given by (with $\tau=t-t^{\prime}$ )

$$
\frac{d G_{i j}(\tau)}{d \tau}=\delta(\tau)\left\langle\left\{S_{i}^{+}, e^{a S_{j}^{z}} S_{j}^{-}\right\}\right\rangle+\left\langle\left\langle\left\{S_{i}^{+}(\tau), \mathcal{H}\right\} ; e^{a S_{j}^{z}} S_{j}^{-}\right\rangle\right\rangle,
$$

which, in the frequency- $\omega$ Fourier space, becomes

$$
\omega G_{i j}(\omega)=i\left\langle\left\{S_{i}^{+}, e^{a S_{j}^{z}} S_{j}^{-}\right\}\right\rangle+i\left\langle\left\langle\left\{S_{i}^{+}(\tau), \mathcal{H}\right\} ; e^{a S_{j}^{z}} S_{j}^{-}\right\rangle\right\rangle_{\omega},
$$

with $G_{i j}(\omega)=\left\langle\left\langle S_{i}^{+}(\tau) ; e^{a S_{j}^{z}} S_{j}^{-}\right\rangle\right\rangle_{\omega}$ and $\langle\langle A(\tau) ; B\rangle\rangle_{\omega}=$ $\int_{-\infty}^{+\infty} d \tau e^{i \omega \tau}\langle\langle A(\tau) ; B\rangle\rangle$. From the basic Poisson brackets (2), a simple algebra yields

$$
i\left\langle\left\{S_{i}^{+}, e^{a S_{j}^{z}} S_{j}^{-}\right\}\right\rangle=\psi(a) \delta_{i j},
$$

where

$$
\psi(a)=-a \Omega(a)+2 \Omega^{\prime}(a)+a \Omega^{\prime \prime}(a),
$$

with

$$
\Omega(a)=\left\langle e^{a S_{i}^{z}}\right\rangle .
$$

Notice that, for the magnetization per spin $m=\left\langle S_{i}^{z}\right\rangle$, the relevant exact relation is fulfilled:

$$
m=\frac{1}{2} \psi(0)=\Omega^{\prime}(0) .
$$

On the other hand, in (6), we have also

$$
\left\{S_{i}^{+}, \mathscr{H}\right\}=i \sum_{h}\left[J_{i h}^{\perp} S_{i}^{z} S_{h}^{+}-J_{i h}^{\|} S_{i}^{+} S_{h}^{z}\right]-i h S_{i}^{+} .
$$

Then, (6) becomes (again without approximations)

$$
\begin{aligned}
(\omega-h) G_{i j}(\omega)= & \psi(a) \delta_{i j} \\
& -\sum_{h}\left[J_{i h}^{\perp}\left\langle\left\langle S_{i}^{z}(\tau) S_{h}^{+}(\tau) ; e^{a S_{j}^{z}} S_{j}^{-}\right\rangle\right\rangle_{\omega}\right. \\
& \left.\quad-J_{i h}^{\|}\left\langle\left\langle S_{i}^{+}(\tau) S_{h}^{z}(\tau) ; e^{a S_{j}^{z}} S_{j}^{-}\right\rangle\right\rangle_{\omega}\right] .
\end{aligned}
$$

The next step consists in performing an appropriate decoupling to close (12). Here we will use the classical version of the Tyablikov decoupling (TD) which, for the quantum case, has been proven [28] to give near-exact results close to the QCP. This decoupling procedure consists in neglecting transverse correlations in (12) so that one can assume that

$$
\begin{aligned}
\left\langle\left\langle S_{h}^{z}(\tau) S_{k}^{+}(\tau) ; e^{a S_{j}^{z}} S_{j}^{-}\right\rangle\right\rangle_{\omega} & \simeq\left\langle S_{h}^{z}\right\rangle\left\langle\left\langle S_{k}^{+}(\tau) ; e^{a S_{j}^{z}} S_{j}^{-}\right\rangle\right\rangle_{\omega} \\
& \simeq m\left\langle\left\langle S_{k}^{+}(\tau) ; e^{a S_{j}^{z}} S_{j}^{-}\right\rangle\right\rangle_{\omega},
\end{aligned}
$$

providing

$$
\left[\omega-h-m J^{\|}(0)\right] G_{i j}(\omega)=\psi(a) \delta_{i j}-m \sum_{h} J_{i h}^{\perp} G_{h j}(\omega),
$$

where $\sum_{h} J_{i h}^{\|}=J^{\|}(0)$ is the $(\mathbf{k}=0)$-component of the $\mathbf{k}$-wave vector Fourier transform in the first Brillouin zone (1BZ) $J_{i j}^{\|}=(1 / N) \sum_{\mathbf{k}} e^{i \mathbf{k} \cdot\left(\mathbf{r}_{i}-\mathbf{r}_{j}\right)} J^{\|}(\mathbf{k})$. Finally, using the Fourier transforms in the $\mathbf{k}$-space

$$
\begin{gathered}
G_{i j}(\omega)=\frac{1}{N} \sum_{\mathbf{k}} e^{i \mathbf{k} \cdot\left(\mathbf{r}_{i}-\mathbf{r}_{j}\right)} G_{\mathbf{k}}(\omega), \\
J_{i j}^{\alpha}=\frac{1}{N} \sum_{\mathbf{k}} e^{i \mathbf{k} \cdot\left(\mathbf{r}_{i}-\mathbf{r}_{j}\right)} J^{\alpha}(\mathbf{k}), \quad \alpha=\perp, \|,
\end{gathered}
$$

with $\delta_{i j}=(1 / N) \sum_{\mathbf{k}} e^{i \mathbf{k} \cdot\left(\mathbf{r}_{i}-\mathbf{r}_{j}\right)}$, (14) reduces to an algebraic equation for $G_{\mathbf{k}}(\omega)$ with solution

$$
G_{\mathbf{k}}(\omega)=\frac{\psi(a)}{\omega-\omega_{\mathbf{k}}},
$$

where

$$
\omega_{\mathbf{k}}=h+m\left[J^{\|}(\mathbf{0})-J^{\perp}(\mathbf{k})\right] .
$$

This equation represents the dispersion relation, at Tyablikov-like decoupling (TD) level, of undamped oscillations for the PFM, expressed as a function of $\mathbf{k}$ in terms of the Fourier transform $J^{\perp}(\mathbf{k})$ of the transverse exchange interaction $J_{i j}^{\perp}$. The key step is to determine the function $\psi(a)$ or $\Omega(a)$ and hence $m=(1 / 2) \psi(0)=\Omega^{\prime}(0)$. For utility of the reader, we outline here in after the classical version of the Callen procedure used to solve this problem for isotropic quantum [35] and classical [34] Heisenberg FMs and for QPFM [28].

From the expression (16) for $G_{\mathbf{k}}(\omega)$ and the exact relation between $G_{\mathbf{k}}(\omega)$ and the corresponding spectral density $\Lambda_{\mathbf{k}}(\omega)$ (see Appendix A)

$$
\Lambda_{\mathbf{k}}(\omega)=i\left[G_{\mathbf{k}}(\omega+i \epsilon)-G_{\mathbf{k}}(\omega-i \epsilon)\right]_{\epsilon \rightarrow 0^{+}},
$$

one easily finds

$$
\Lambda_{\mathbf{k}}(\omega)=2 \pi \psi(a) \delta\left(\omega-\omega_{\mathbf{k}}\right) .
$$

Then, the spectral density $\Lambda_{i j}(\omega)$ corresponding to $G_{i j}(\omega)$ can be obtained via its Fourier transform:

$$
\begin{aligned}
\Lambda_{i j}(\omega) & =\frac{1}{N} \sum_{\mathbf{k}} e^{i \mathbf{k} \cdot\left(\mathbf{r}_{i}-\mathbf{r}_{j}\right)} \Lambda_{\mathbf{k}}(\omega) \\
& =2 \pi \psi(a) \frac{1}{N} \sum_{\mathbf{k}} e^{i \mathbf{k} \cdot\left(\mathbf{r}_{i}-\mathbf{r}_{j}\right)} \delta\left(\omega-\omega_{\mathbf{k}}\right) .
\end{aligned}
$$


Now, with these ingredients, we are in position to obtain the correlation function $\langle B A\rangle=\left\langle e^{a S_{j}^{z}} S_{j}^{-} S_{i}^{+}\right\rangle$related to the original GF $G_{i j}(\omega)=\langle\langle A(\tau) ; B\rangle\rangle_{\omega}=\left\langle\left\langle S_{i}^{+}(\tau) ; e^{a S_{j}^{z}} S_{j}^{-}\right\rangle\right\rangle_{\omega}$.

From the classical spectral theorem (see Appendix A, (A.13) and (A.14)), one immediately gets

$$
\left\langle e^{a S_{j}^{z}} S_{j}^{-} S_{i}^{+}\right\rangle=T \int_{-\infty}^{+\infty} \frac{d \omega}{2 \pi} \frac{\Lambda_{i j}(\omega)}{\omega} \simeq T \psi(a) \frac{1}{N} \sum_{\mathbf{k}} \frac{e^{i \mathbf{k} \cdot\left(\mathbf{r}_{i}-\mathbf{r}_{j}\right)}}{\omega_{\mathbf{k}}},
$$

and hence also

$$
\left\langle e^{a S_{i}^{z}} S_{i}^{-} S_{i}^{+}\right\rangle=\Phi \psi(a)
$$

Here, the quantity

$$
\Phi=\frac{T}{N} \sum_{\mathbf{k}} \frac{1}{\omega_{\mathbf{k}}} \stackrel{N \rightarrow{ }^{\infty}}{=} T \int_{1 \mathrm{BZ}} \frac{d^{d} k}{(2 \pi)^{d}} \frac{1}{\omega_{\mathbf{k}}}
$$

is independent of the Callen parameter $a$.

On the other hand, from the relation $S_{i}^{+} S_{i}^{-}=S_{i}^{-} S_{i}^{+}=$ $S^{2}-\left(S_{i}^{z}\right)^{2}$, we can also write

$$
\left\langle e^{a S_{i}^{z}} S_{i}^{-} S_{i}^{+}\right\rangle=S^{2} \Omega(a)-\Omega^{\prime \prime}(a)
$$

Then, combining (22) and (24), where $\psi(a)$ is given by (8), we obtain the following differential equation for $\Omega(a)$ :

$$
\Omega^{\prime \prime}(a)+2\left(\frac{1}{\Phi}+a\right) \Omega^{\prime}(a)-S^{2} \Omega(a)=0
$$

to be solved with the initial condition $\Omega(0)=1$ and the additional one $\Omega(a)=\int_{-S}^{S} d S^{z} f\left(S^{z}\right) e^{a S^{z}}$, arising from the ensemble-average definition of $e^{a S^{z}}$.

With these conditions we have obtained in [34] the physical solution of (25):

$$
\Omega(a)=\frac{S / \Phi}{S / \Phi+a} \frac{\sinh (S / \Phi+a)}{\sinh (S / \Phi)}
$$

This key result (26) represents the classical analogue of the famous Callen formula for quantum spin- $S$ models [35]. An alternative and very instructive method to obtain the solution (26) is presented in Appendix B.

Taking into account the exact relation (10), (26) gives the the remarkable expression:

$$
m=S\left[\operatorname{coth}\left(\frac{S}{\Phi}\right)-\frac{\Phi}{S}\right]=S L\left(\frac{S}{\Phi}\right)
$$

which is valid for any $d, T$, and $h$. Here, $L(x)=\operatorname{coth} x-1 / x$ is the well-known Langevin function and $\Phi$ is expressed by (23) in terms of the dispersion relation $\omega_{\mathbf{k}}$. If we use the TD, $\omega_{\mathbf{k}}$ is given by (17) which is in turn a function of $m$. Hence, (27) is a self-consistent equation for $m$ and $T$. The longitudinal magnetic field $h$ and the anisotropy enter into the problem via the function $\Phi$.

Of course, in the spirit of the Callen method for the calculation of $m$, it is possible to introduce more elaborate decoupling procedures which preserve its validity.
For instance, we could adopt the Callen decoupling (CD) [35], with its classical variant [34],

$$
\left\langle\left\langle S_{h}^{z}(\tau) S_{k}^{+}(\tau) ; e^{a S_{j}^{z}} S_{j}^{-}\right\rangle\right\rangle_{\omega} \simeq m\left[G_{k j}(\omega)-\frac{\left\langle S_{h}^{-} S_{k}^{+}\right\rangle}{2 S^{2}} G_{h j}(\omega)\right],
$$

which takes into account the transverse correlations to the leading order and implies the dispersion relation:

$$
\omega_{\mathbf{k}}^{(\mathrm{CD})}=\omega_{\mathbf{k}}^{(\mathrm{TD})}+\frac{m}{2 S^{2}} \frac{1}{N} \sum_{\mathbf{k}^{\prime}}\left[J^{\perp}\left(\mathbf{k}^{\prime}\right)-J^{\|}\left(\mathbf{k}-\mathbf{k}^{\prime}\right)\right] C\left(\mathbf{k}^{\prime}\right)
$$

with

$$
C(\mathbf{k})=\sum_{i} e^{-i \mathbf{k} \cdot\left(\mathbf{r}_{i}-\mathbf{r}_{j}\right)}\left\langle S_{i}^{+} S_{j}^{-}\right\rangle .
$$

However, in the present case, the TD and the CD (28) provide essentially identical results close to the $(T=0)$-critical point where $m$ is near the full polarized-state value $S$.

Given the magnetization $m$, the thermodynamics of our CPFM will be derived using the general formalism of the classical two-time Green functions (see Appendix A and [33]).

\section{Transverse Susceptibility and Critical Line}

We have now all the elements to extract the physics of interest setting $a=0$ in the previous results and solving the set of self-consistent equations:

$$
\begin{gathered}
\sigma=\operatorname{coth}\left(\frac{S}{\Phi}\right)-\frac{\Phi}{S}=L\left(\frac{S}{\Phi}\right), \\
\Phi=T \int_{1 \mathrm{BZ}} \frac{d^{d} k}{(2 \pi)^{d}} \frac{1}{\omega_{\mathbf{k}}(\sigma)}, \\
\omega_{\mathbf{k}}(\sigma)=\omega_{0}(\sigma)+\sigma S\left[J^{\perp}(0)-J^{\perp}(\mathbf{k})\right] \geq 0,
\end{gathered}
$$

where $\omega_{0}(\sigma)=h+S \sigma\left[J^{\|}(0)-J^{\perp}(0)\right]$. Here we have conveniently introduced the reduced magnetization per spin $\sigma=m / S$, with $0 \leq \sigma \leq 1$. Equations (31) will give $\sigma$ as a function of $T$ and $h$ and hence the GF (16), also at $a=0$.

For our aim, the relevant quantity to be calculated is the transverse GF:

$$
G_{\perp}(\mathbf{k}, \omega)=\left.\left\langle\left\langle S_{i}^{+}(\tau) ; S_{j}^{-}\right\rangle\right\rangle_{\mathbf{k}, \omega} \equiv G_{\mathbf{k}}(\omega)\right|_{a=0} .
$$

This allows to determine the dynamical transverse susceptibility for CPFM:

$$
\chi_{\perp}(\mathbf{k}, \omega)=-G_{\perp}(\mathbf{k}, \omega) \equiv-\left.G_{\mathbf{k}}(\omega)\right|_{a=0},
$$

where, at the TD level,

$$
G_{\perp}(\mathbf{k}, \omega)=\frac{2 S \sigma}{\omega-\omega_{\mathbf{k}}(\sigma)} .
$$

In particular, the thermodynamic transverse susceptibility is given by

$$
\chi_{\perp}(T, h) \equiv \chi_{\perp}(\mathbf{k}=\mathbf{0}, \omega=0)=\frac{2 S \sigma}{\omega_{0}(\sigma)}=\frac{2 S \sigma(T, h)}{h+S \sigma\left[J^{\|}(0)-J^{\perp}(0)\right]} .
$$


Using (35) it is also possible to obtain the transverse correlation length via the following relation [36]:

$$
\xi_{\perp}^{2}=-\frac{1}{2} \chi_{\perp}^{-1}(0,0)\left[\frac{d^{2}\left(\chi_{\perp}(\mathbf{k}, 0)\right)}{d \mathbf{k}^{2}}\right]_{\mathbf{k}=0},
$$

where $\chi_{\perp}(\mathbf{k}, 0)=2 S \sigma / \omega_{\mathbf{k}}$. The longitudinal susceptibility will be simply given by $\chi_{\|}(T, h)=S(\partial \sigma(T, h) / \partial h)$. Of course, the stability condition $\chi_{\perp} \geq 0$ requires that, in (35), the inequality $\omega_{0}(\sigma)=h+S \sigma(T, h)\left[J^{\|}(0)-J^{\perp}(0)\right] \geq 0$ must be fulfilled. The equality is physically possible for $h>0$ and $\sigma>$ 0 only if $J^{\|}(0)<J^{\perp}(0)$, which is the regime characterizing the CPFM of interest to us.

Here in after we will focus on quantum-like criticality related to the field-driven easy-plane ordering whose key quantities are $\omega_{0}$ and $\chi_{\perp}$ and the related ones as functions of $T$ and $h$. However, in some relevant cases which may have physical interest, we will calculate also the longitudinal quantities $\sigma(T, h)$ and $\chi_{\|}(T, h)$. Other expressions can be obtained by means of known thermodynamic relations [33].

We start exploring the main features of the CPFM phase diagram in the $(h, T)$-plane. These results will be used as a basis for next developments. At zero temperature with $h \neq 0$, (31) provides the solution $\sigma=1$ for the reduced magnetization, characterizing a fully polarized state. This implies that the dispersion relation is given by $\omega_{\mathbf{k}}=\omega_{0}+$ $S\left[J^{\perp}(0)-J^{\perp}(\mathbf{k})\right]$, where $\omega_{0}=h-S\left[J^{\perp}(0)-J^{\|}(0)\right]$ is the frequency gap. Hence the transverse susceptibility, which has physical meaning for $h \geq S\left[J^{\perp}(0)-J^{\|}(0)\right]>0$, becomes

$$
\chi_{\perp}=\frac{2 S}{h-S\left[J^{\perp}(0)-J^{\|}(0)\right]} .
$$

Remarkably, (37) suggests that, despite the classical nature of our anisotropic spin model, there exists, as in the quantum case [26-28], a $(T=0)$-critical point at the value $h_{c}=$ $S\left[J^{\perp}(0)-J^{\|}(0)\right]$ of the longitudinal magnetic field. Then, crossing this point, decreasing $h$ to $h_{c}$, a field-induced second-order $(T=0)$-phase transition arises from a fully polarized state with $\sigma=1$ to a transverse-ordered phase. However, the latter phase is unaccessible by the present analysis due to the absence in the Hamiltonian (1) of an inplane symmetry breaking magnetic field.

From (37), with $\omega_{0}=h-h_{c} \geq 0$, we have $\chi_{\perp}=$ $2 S\left(h-h_{c}\right)^{-1}$ as $h \rightarrow h_{c}^{+}$, defining the mean field exponent $\gamma_{h}=1$. (Through the paper we will use the indices $h$ and $T$ to denote the horizontal (isothermal) and the vertical trajectories approaching a critical point in the $(h, T)$-plane, resp.)

For arbitrary temperature we can write $\omega_{0}(\sigma)=h-\sigma h_{c} \geq$ 0 (so that $\left.\omega_{\mathbf{k}}=\left(h-\sigma h_{c}\right)+S \sigma\left[J^{\perp}(0)-J^{\perp}(\mathbf{k})\right]\right)$. Hence the transverse susceptibility can be conveniently written as

$$
\chi_{\perp}(T, h)=\frac{2 S \sigma(T, h)}{h-\sigma(T, h) h_{c}},
$$

with $h \geq \sigma(T, h) h_{c}$ or $\sigma(T, h) \leq h / h_{c}$ for stability reasons. Equation (38) establishes an interesting relation between the transverse physics and the longitudinal one.
In the $(h, T)$-plane, where $\chi_{\perp}=\infty\left(\omega_{0}(\sigma)=0\right)$, the general equation which determines the possible critical points is

$$
h-\sigma(T, h) h_{c}=0,
$$

or, in view of the equation for $\sigma$,

$$
\frac{h}{h_{c}}=L\left(\frac{S}{\Phi_{c}}\right) \text {. }
$$

Here,

$$
\Phi_{c}=\Phi\left(T, h ; \sigma=\frac{h}{h_{c}}\right)=\frac{T}{J^{\perp}(0) S} \frac{h_{c}}{h} F_{d}(-1),
$$

where $\omega_{\mathbf{k}}^{(c)}=\omega_{\mathbf{k}}\left(\sigma=h / h_{c}\right)=S\left(h / h_{c}\right) J^{\perp}(0)\left(1-\gamma_{\mathbf{k}}^{\perp}\right)$ at the critical points and

$$
F_{d}(-1)=\int_{1 \mathrm{BZ}} \frac{d^{d} k}{(2 \pi)^{d}} \frac{1}{\left(1-\gamma_{\mathrm{k}}^{\perp}\right)},
$$

with $\gamma_{\mathbf{k}}^{\perp}=J^{\perp}(\mathbf{k}) / J^{\perp}(0) \leq 1$. More explicitly, (40) can be also written as

$$
h+F_{d}(-1) \frac{T}{J^{\perp}(0) S^{2}} \frac{h_{c}^{2}}{h}-h_{c} \operatorname{coth}\left[\left(F_{d}(-1) \frac{T}{J^{\perp}(0) S^{2}} \frac{h_{c}}{h}\right)^{-1}\right]=0 \text {. }
$$

The quantity $F_{d}(-1)$ is one of the so-called structure sums $F_{d}(n)=(1 / N) \sum_{\mathbf{k}}\left(1-\gamma_{\mathbf{k}}^{\perp}\right)^{n} \stackrel{N \rightarrow \infty}{=} \int_{1 \mathrm{BZ}}\left(d^{d} k /(2 \pi)^{d}\right)\left(1-\gamma_{\mathbf{k}}^{\perp}\right)^{n}$ depending only on the lattice structure of the spin model. Accurate numerical values of $F_{d}(n)$ can be found in the literature for different $d$ and lattice structures [34, 35, 37]. Previous results suggest that, while a $(T=0)$-CP with $\sigma=1$ exists for any $d$, a critical line with $0 \leq \sigma \leq 1$, ending in such a point, may occur only for dimensionalities for which the integral (42) converges.

If we consider short-range interactions and an hypercubic lattice $J^{\alpha}(\mathbf{k})=2 J^{\alpha} \sum_{\nu=1}^{d} \cos k_{v} \simeq J^{\alpha}(0)-J^{\alpha} k^{2}(\alpha=\perp$, $\|)$ as $k \rightarrow 0$ with $J^{\alpha}(0)=2 d J^{\alpha}$, from (42) it immediately follows that for $d \leq 2$ only the ( $T=0)$-CP exists, while for $d>2$ a finite-temperature critical line, ending in the $(T=0)$-CP $\left(h_{c}, T=0\right)$, occurs consistently with the Mermin-Wagner theorem [38]. The same result has been obtained in the quantum case [28].

For $d>2$, the critical line equation (42) (or (43)) can be solved numerically with respect to $h$ or $T$ providing the representation $h_{c}(T)$ or $T_{c}(h)$. Notice that, along the critical line, the reduced magnetization $\sigma\left(T, h_{c}(T)\right)=\sigma(T)$ is simply given by $\sigma(T)=h_{c}(T) / h_{c}$ when $h_{c}(T)$ is known. The critical line in the plane $(h, T)$ for $d=3$ is plotted in Figure 1.

Starting from (40) or (43) we can easily derive the analytical expression of the zero-field critical temperature $T_{c}(h=$ $0) \equiv T_{c}$ where $\sigma=0$ irrespective of the specific structure of $\gamma_{\mathbf{k}}^{\perp}$ in (37) (as in the case of short-range interactions for which $\gamma_{\mathbf{k}}^{\perp}=J^{\perp}(\mathbf{k}) / J^{\perp}(0)=\left(2 J^{\perp} / J^{\perp}(0)\right) \sum_{\nu=1}^{d} \cos k_{\nu}=$ $\left.(1 / d) \sum_{\nu=1}^{d} \cos k_{\nu}\right)$.

From the expansion coth $x \simeq 1 / x+(1 / 3) x+O\left(x^{3}\right)$, and hence $L(x)=\operatorname{coth} x-1 / x \simeq x / 3+O\left(x^{3}\right)$, for $x=S / \Phi_{c} \ll 1$, (40) provides, for $\Phi_{c} \rightarrow \infty$ as $h \rightarrow 0$ at finite $T$ (see (41)),

$$
\frac{T_{c}}{J^{\perp}(0) S^{2}}=\frac{1}{3 F_{d}(-1)} \text {. }
$$




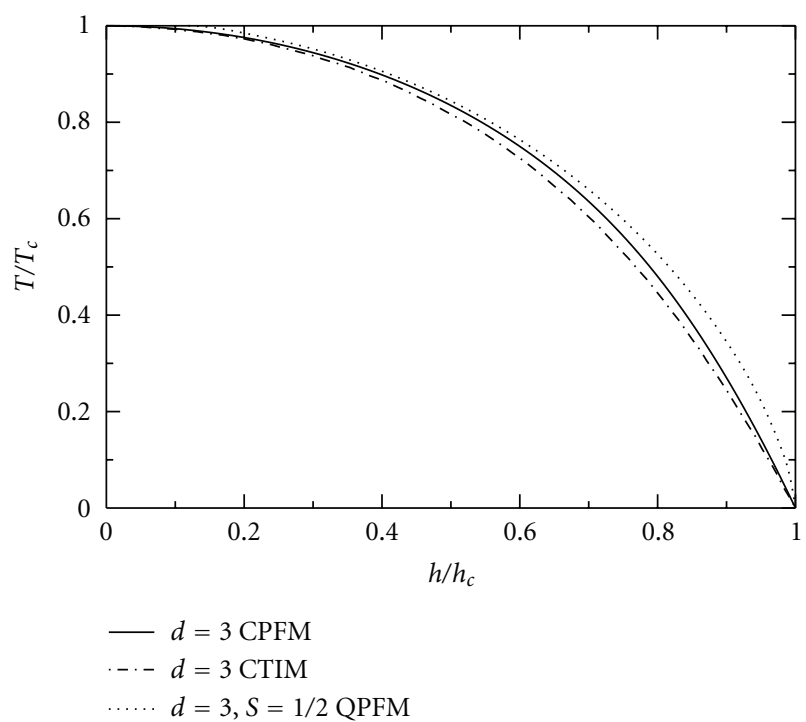

Figure 1: Critical line of the classical planar ferromagnet (CPFM) with short-range interactions on a three-dimensional cubic lattice (present work). Here $T_{c}$ denotes the critical temperature at $h=0$ and $h_{c}$ the critical magnetic field at $T=0$. A comparison is shown with the corresponding curves for the classical transverse Ising model (CTIM), obtained by RG calculations, and for the spin-1/2 quantum planar ferromagnet (QPFM). The critical line for the spin$S$ QPFM with long-range interactions in the classical limit $S \rightarrow \infty$ has been also obtained at the Tyablikov decoupling level, and, as expected, it coincides with the one calculated for the CPFM.

It is worth noting that for QPFM, within the TD it was found that $[28] T_{c}^{\mathrm{QPFM}} / J^{\perp}(0) S(S+1)=1 / 3 F_{d}(-1)$, which reproduces, as expected, (44) in the classical limit for $S \rightarrow \infty$.

For short-range interactions and an hypercubic lattice, estimates for $d>2$ can be obtained assuming $1-\gamma_{\mathrm{k}}^{\perp} \simeq k^{2} / 2 d$ as $k \rightarrow 0$.

Now we explore the behavior of the critical line in the low-temperature regime close to the $(T=0)$-CP where $\Phi_{c} \ll 1$. With $L\left(S / \Phi_{c}\right) \simeq 1-\Phi_{c} / S+2 e^{-2 S / \Phi_{c}},(40)$ becomes

$$
\frac{h}{h_{c}} \simeq 1-F_{d}(-1) \frac{T}{J^{\perp}(0) S^{2}} \frac{h_{c}}{h}+O\left(e^{-\left(2 / F_{d}(-1)\right)\left(J^{\perp}(0) S^{2} / T\right)}\right) \text {. }
$$

Solving the equation with respect to $h$ or $T$, we find

$$
\begin{aligned}
h_{c}(T) \simeq h_{c}\{1- & F_{d}(-1) \frac{T}{J^{\perp}(0) S^{2}} \\
& \left.+O\left(e^{-\left(2 / F_{d}(-1)\right)\left(J^{\perp}(0) S^{2} / T\right)}\right)\right\}, \quad T \longrightarrow 0,
\end{aligned}
$$

or

$$
\begin{aligned}
T_{c}(h) \simeq & \frac{J^{\perp} S^{2}}{F_{d}(-1) h_{c}}\left(h_{c}-h\right) \\
& +O\left(\left(e^{-\left(2\left(F_{d}(-1)\right)^{2} h_{c}\right) /\left(h_{c}-h\right)}\right)\right), \quad h \longrightarrow h_{c}^{-} .
\end{aligned}
$$

Along this branch of the critical line we have also

$$
\sigma(T) \simeq 1-F_{d}(-1) \frac{T}{J^{\perp}(0) S^{2}}+O\left(e^{-\left(2 / F_{d}(-1)\right)\left(J^{\perp}(0) S^{2} / T\right)}\right) .
$$

From the low-temperature representations (46) and (47) for the critical line we can extract the shift exponent $\psi=1$, which determines the shape of the phase boundary close to the $(T=0)$-CP. It has to be stressed that the value of $\psi$ is independent of $d$, in contrast with the result $(\psi=d / 2)$ known for the QPFM $[27,28]$.

\section{Low-Temperature Critical Properties}

In this section we study the low-temperature properties and crossovers of our CPFM within the easy-plane-disordered phase, close to the field-induced ( $T=0$ )-CP where $\sigma \approx 1$, $h \simeq h_{c}$ (nearly polarized state), and $\omega_{0}(\sigma)=h-\sigma h_{c}$ is very small. Under these conditions, in $(31) \Phi(\sigma) / S \ll 1$; therefore, the equation for $\sigma$ becomes

$$
\sigma \simeq 1-\frac{\Phi(\sigma)}{S}+2 e^{-(2 S / \Phi(\sigma))}
$$

The quantity $\Phi(\sigma) \equiv \Phi\left(\omega_{0}\right)$ near criticality can be suitably estimated assuming, for the oscillation spectrum $\omega_{\mathrm{k}}$, the low$\mathbf{k}$ expression $\omega_{\mathbf{k}} \simeq \omega_{0}+S \sigma J^{\perp} k^{2}$. This provides

$$
\Phi \simeq \frac{d}{2}\left(\frac{T}{\omega_{0}}\right) \int_{0}^{1} d x \frac{x^{d / 2-1}}{1+\left(\tau / \omega_{0}\right)}
$$

where $\tau \approx S J^{\perp} \Lambda_{1 \mathrm{BZ}}^{2}=\left(S J^{\perp}(0) / 2 d\right) \Lambda_{1 \mathrm{BZ}}^{2}$ and $\Lambda_{1 \mathrm{BZ}}$ is a natural wave-vector cut-off related to the first Brillouin zone and determined by $(1 / N) \sum_{\mathbf{k}}=1$ or $($ as $N \rightarrow \infty) \Lambda_{1 \mathrm{BZ}}=$ $\left(d / K_{d}\right)^{1 / d}$, with $K_{d}=2^{1-d} \pi^{-d / 2} / \Gamma(d / 2)$.

As a consequence, to the leading order in $\Phi$, the selfconsistent equation for $\sigma$ can be written as (except for exponentially small terms)

$$
\sigma \simeq 1-\frac{1}{S}\left(\frac{T}{\omega_{0}(\sigma)}\right) F\left(1, \frac{d}{2} ; \frac{d}{2}+1 ;-\frac{\tau}{\omega_{0}(\sigma)}\right)
$$

where $F(\alpha, \beta ; \gamma ; z)$ is the hypergeometric function.

For our purposes, it is convenient to transform (51) for the longitudinal physics into a self-consistent equation for the oscillation gap $\omega_{0}$, strictly related to $\chi_{\perp}$ and hence to the transverse physics.

Since $\sigma=h / h_{c}-\omega_{0} / h_{c}$, straightforward calculations provide the following expression for (51) in terms of the natural variable $\omega_{0} / T$ :

$$
\frac{\omega_{0}}{T}=\frac{g}{T}+\frac{h_{c}}{S \tau}\left(\frac{\tau}{\omega_{0}}\right) F\left(1, \frac{d}{2} ; \frac{d}{2}+1 ;-\frac{\tau}{\omega_{0}}\right) .
$$

Here $g=h-h_{c}$ and $\omega_{0} / T=2 S \sigma / T \chi_{\perp} \simeq 2 S / T \chi_{\perp} \propto$ $\left(T \chi_{\perp}\right)^{-1} \propto\left(T \xi_{\perp}^{2}\right)^{-1}$. Notice that since $\chi_{\perp}(\mathbf{k}, 0) \simeq 2 S \sigma /\left[\omega_{0}+\right.$ $\left.\sigma S J^{\perp} \mathbf{k}^{2}\right]$, from $(37)$, one gets $\xi_{\perp} \simeq J^{\perp} \chi_{\perp}^{1 / 2}$.

Of course, once $\omega_{0}$ has been determined as a function of $(T, h)$, one can directly calculate $\chi_{\perp}(T, h)$ and, using the relation between $\omega_{0}$ and $\sigma$, it is possible to determine $\sigma(T, h)$ and, therefore, the longitudinal physics near the polarized state in the low-temperature critical regime. 
Adopting the asymptotic expansions of the hypergeometric function $F(1, v ; \nu+1 ;-(1 / z))$ for $z \ll 1$,

$$
F\left(1, v ; v+1 ;-\frac{1}{z}\right) \approx \begin{cases}\frac{\pi v}{\sin \pi \nu} z^{\nu}, & v<1, \\ z \ln \frac{1}{z}, & v=1, \\ \frac{v}{v-1} z-\frac{\pi v}{|\sin \pi v|} z^{v}, & 1<v<2,\end{cases}
$$

now, we can systematically explore the thermodynamics of the CPFM close to the $(T=0)$-CP for different values of the dimensionality $d$ of the system. Bearing this in mind, in strict analogy with the quantum case $[28,39]$, in the following subsections we will show the asymptotic solutions of (52) in the classical $\omega_{0} / T \ll 1\left(\Leftrightarrow T \xi_{\perp}^{2} \gg 1\right.$ or $\left.T \chi_{\perp} \gg 1\right)$ and quantum-like $\left(\omega_{0} / T \gg 1 \Leftrightarrow T \xi_{\perp}^{2} \ll 1\right.$ or $\left.T \chi_{\perp} \ll 1\right)$ regimes, respectively, and the estimates of the related crossovers for different values of $d$.

4.1. $d<2$. Replacing the expansions (53) in the self-consistent equation (52) for $\omega_{0}$, we get

$$
\frac{\omega_{0}}{T} \simeq \frac{g}{T}+\frac{\pi d / 2}{\sin (\pi d / 2)} \frac{h_{c}}{S \tau}\left(\frac{T}{\tau}\right)^{(d-2) / 2}\left(\frac{\omega_{0}}{T}\right)^{(d-2) / 2},
$$

In the regime $\omega_{0} / T \ll 1\left(T \xi_{\perp}^{2} \gg 1\right)$, classical regime in the quantum critical scenario near the isolated ( $T=0)-\mathrm{CP},(54)$ admits a solution only for $h<h_{c}$, which reads

$$
\omega_{0} \simeq\left[\frac{\pi d / 2}{\sin (\pi d / 2)} \frac{h_{c}}{S \tau^{d / 2}}\right]^{2 /(2-d)}\left(\frac{T}{h_{c}-h}\right)^{2 /(2-d)} .
$$

Under the consistency condition $T \ll\left(h_{c}-h\right)^{2 / d}$. Then, for $h<h_{c}$ within the region $T \ll\left(h_{c}-h\right)^{2 / d}$, in the $(h, T)$-plane, the transverse and longitudinal susceptibilities are given by

$$
\begin{gathered}
\chi_{\perp} \simeq 2 S\left[\frac{\pi d / 2}{\sin (\pi d / 2)} \frac{h_{c}}{S \tau^{d / 2}}\right]^{-2 /(2-d)}\left(\frac{T}{h_{c}-h}\right)^{-2 /(2-d)}, \\
\chi_{\|} \simeq \frac{S}{h_{c}}\left\{1-\left[\frac{\pi d / 2}{\sin (\pi d / 2)} \frac{h_{c}}{S \tau^{d / 2}}\right]^{2 /(2-d)}\left(\frac{h_{c}}{h_{c}-h}\right)^{2 /(2-d)}\right. \\
\left.\times\left(\frac{T}{h_{c}-h}\right)^{2 /(2-d)}\right\},
\end{gathered}
$$

where the reduced magnetization near polarization is immediately given as $\sigma(T, h) \simeq 1-\omega_{0} / h_{c}$.

It is worth noting that, for fixed $h<h_{c}$ and $T \rightarrow 0$, the transverse susceptibility diverges with the critical exponent $\gamma_{T}=2 /(2-d)$, while the longitudinal one remains finite.

In the regime $\omega_{0} / T \gg 1\left(T \xi_{\perp}^{2} \ll 1\right)$, named here quantum-like regime again in analogy with the corresponding quantum scenario, different terms in (54) may enter in competition and different asymptotic behaviors are expected to occur close to the isolated $(T=0)$-CP. For $h<h_{c}$ in the region $\left(h_{c}-h\right)^{2 / d} \ll T \ll\left(h_{c}-h\right)^{(4-d) / 2}$, where $1 \ll$ $\omega_{0} / T \ll g / T$, (54) provides, to the leading order, a solution which is formally identical to (55) but now the condition $\omega_{0} / T \gg 1$ should hold. The same occurs for the thermodynamic quantities (56) and the related ones.

For $h=h_{c}$, decreasing $T$ along a vertical trajectory which corresponds to the quantum critical one in the phase diagram of the QPFM [28], (54) yields, with $\omega_{0}\left(T, h_{c}\right) \equiv$ $\omega_{0 c}(T)$,

$$
\omega_{0 c}(T) \simeq \tau\left[\frac{\pi d / 2}{\sin (\pi d / 2)} \frac{h_{c}}{S \tau}\right]^{2 /(4-d)}\left(\frac{T}{\tau}\right)^{2 /(4-d)} .
$$

Then, we get

$$
\chi_{\perp}\left(T, h_{c}\right) \simeq 2\left(\frac{S}{\tau}\right)\left[\frac{\pi d / 2}{\sin (\pi d / 2)} \frac{h_{c}}{S \tau}\right]^{-2 /(4-d)}\left(\frac{T}{\tau}\right)^{-2 /(4-d)},
$$

which defines the critical exponent $\gamma_{T}=2 /(4-d)$, with $1 / 2<$ $\gamma_{T}<1$. For the nearly polarized state (with $\sigma(T, h) \lesssim 1$ ), a simple algebra yields

$$
\sigma\left(T, h_{c}\right) \simeq 1-\frac{\tau}{h_{c}}\left[\frac{\pi d / 2}{\sin (\pi d / 2)} \frac{h_{c}}{\tau}\right]^{2 /(4-d)}\left(\frac{T}{\tau}\right)^{2 /(4-d)},
$$

which increases towards unity decreasing $T$ according to the power-law $\sim T^{\gamma_{T}}$.

Now we consider the region which is more relevant from the experimental point of view, namely, the $\mathrm{V}$-shaped region $T \gg\left|h-h_{c}\right|^{(4-d) / 2}$, around the vertical trajectory $h=h_{c}$ (for both $h \lesssim h_{c}$ and $h \gtrsim h_{c}$ ). Under this condition, from (54) a straightforward algebra gives

$$
\omega_{0}(T, h) \simeq \omega_{0 c}(T)\left\{1+\frac{2}{4-d} \frac{h-h_{c}}{\omega_{0 c}(T)}\right\} .
$$

This expression suggests that, within the V-shaped region, the thermodynamics is essentially identical to the one along the trajectory $h=h_{c}$, except for a small correction $\sim\left|h-h_{c}\right|$.

Finally, for $h>h_{c}$ and sufficiently far from the quantumlike critical trajectory, within the region $T \ll\left(h-h_{c}\right)^{(4-d) / 2}$, we have

$$
\omega_{0}(T, h) \simeq\left(h-h_{c}\right)\left\{1+\frac{\pi d / 2}{\sin (\pi d / 2)} \frac{h_{c}}{S \tau^{d / 2}} \frac{T}{\left(h-h_{c}\right)^{(4-d) / 2}}\right\} .
$$

This implies that

$$
\chi_{\perp}(T, h) \simeq 2 S\left(h-h_{c}\right)^{-1}\left\{1-\frac{\pi d / 2}{\sin (\pi d / 2)} \frac{h_{c}}{S \tau^{d / 2}} \frac{T}{\left(h-h_{c}\right)^{(4-d) / 2}}\right\},
$$

which differs from the MF result $\chi_{\perp} \simeq 2 S\left(h-h_{c}\right)^{-1}$, found before at $T=0$, for a small power-law correction in temperature, in contrast with the exponentially small correction which occurs in the quantum counterpart [28]. Besides, for the nearly polarized state, we obtain

$$
\begin{aligned}
\sigma(T, h) & \simeq 1-\frac{\pi d / 2}{\sin (\pi d / 2)} \frac{1}{S \tau^{d / 2}} \frac{T}{\left(h-h_{c}\right)^{(2-d) / 2}}, \\
\chi_{\|}(T, h) & \simeq \frac{2-d}{2} \frac{\pi d / 2}{\sin (\pi d / 2)} \frac{1}{\tau^{d / 2}} \frac{T}{\left(h-h_{c}\right)^{(4-d) / 2}} .
\end{aligned}
$$




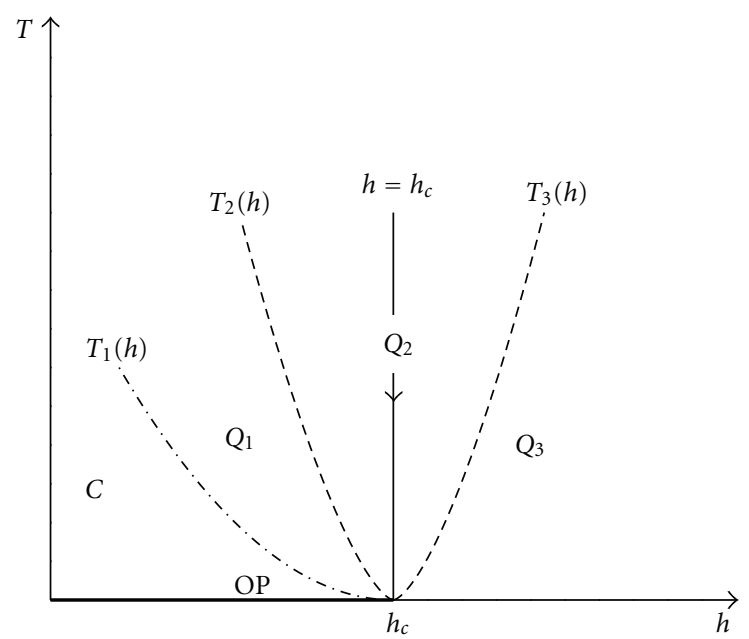

FIGURE 2: Qualitative phase diagram of CPFM with short-range interactions for $d<2$, close to the zero-temperature critical point. The line $T_{1}(h)$ marks the crossover between the regimes $T \chi_{\perp} \propto$ $T \xi_{\perp}^{2} \gg 1($ region $C)$ and $T \chi_{\perp} \propto T \xi_{\perp}^{2} \ll 1\left(\right.$ region $Q \equiv\left(Q_{1}, Q_{2}, Q_{3}\right)$ ) increasing $h$. The lines $T_{2}(h)$ and $T_{3}(h)$, symmetric with respect to the vertical line $h=h_{c}$, signal the crossovers among the subregimes $Q_{1}, Q_{2}$, and $Q_{3}$. Finally, the heavy line OP represents the zerotemperature-ordered phase.

In summary, the previous results suggest, for $d<2$, a very rich phase diagram around the isolated $(T=0)$ $\mathrm{CP}$, qualitatively reported in Figure 2, where different low- $T$ regimes and crossover lines are presented. It appears divided in two main regions (named in analogy with the quantum case [28]): $C$, where $\omega_{0} / T \ll 1\left(T \chi_{\perp} \propto T \xi_{\perp}^{2} \gg 1\right)$, and $Q \equiv$ $\left(Q_{1}, Q_{2}, Q_{3}\right)$, where $\omega_{0} / T \gg 1\left(T \xi_{\perp}^{2} \ll 1\right)$. The line $T_{1} \simeq$ $\left(h_{c}-h\right)^{2 / d}$ for $h<h_{c}$ signals the crossover between the regimes $C$ and $Q$; the lines $T_{2}(h) \sim\left(h_{c}-h\right)^{(4-d) / 2}$ and $T_{3}(h) \sim\left(h-h_{c}\right)^{(4-d) / 2}$, symmetric to the vertical trajectory $h=h_{c}$, provide the signature of crossovers among three distinct subregimes $Q_{1}, Q_{2}$, and $Q_{3}$ with different asymptotic behaviors of the thermodynamic quantities as functions of $T$ and $h$. We stress that, within the $V$-shaped region $Q_{2}$, delimited by the crossover lines $T_{2}(h)$ and $T_{3}(h)$, the $T$ dependent behaviors are essentially identical to those along the trajectory $h=h_{c}$ except for different a small power law corrections $\sim\left|h-h_{c}\right|$. Besides, in the regime $Q_{3}$, decreasing $T$ at fixed $h>h_{c}$, the transverse susceptibility deviates from the one at $T=0$ except for a small power law correction as a function of $T$ and $h-h_{c}$. The latter feature differs crucially from the QPFM scenario where the correction to the $(T=0)$-behavior of $\chi_{\perp}$ is an exponentially small function of $T$ and $h-h_{c}[28,39]$.

In any case, below two dimensions, the global phase diagram and the crossovers of the CPFM and QPFM are quite similar. This similarity represents a very interesting ingredient for experimental studies in the sense specified in the introductory section.

4.2. $d=2$. For the two-dimensional CPFM, which also exhibits only a $(T=0)$-CP, a low-temperature scenario similar to the one derived for $d<2$ takes place. But now, logarithmic corrections to the leading power-law behavior arise. This peculiarity has been also found for the analogous quantum system [28].

With the expansion (53), close to the $(T=0)$-CP the general self-consistent equation (52) reduces to

$$
\frac{\omega_{0}}{T} \simeq \frac{g}{T}+\frac{h_{c}}{S \tau} \ln \left(\frac{\tau}{\omega_{0}}\right) .
$$

First, we consider the regime $\omega_{0} / T \ll 1$. If $h \geq h_{c}$, no solution exists while, for $h<h_{\mathcal{c}}$, (64) provides

$$
\omega_{0} \simeq \tau \exp \left(-\frac{S \tau}{h_{c}} \frac{h_{c}-h}{T}\right),
$$

which has to be compared with the corresponding result achieved for the QPFM, $\omega_{0} \simeq T e^{-\left(S \tau / h_{c}\right)\left(\left(h_{c}-h\right) / T\right)}$ [28]. Then, for the transverse susceptibility we have the exponentially divergent behavior $\chi_{\perp} \simeq(2 S / \tau) \exp \left[\left(S \tau / h_{c}\right)\left(\left(h_{c}-h\right) / T\right)\right]$ as $T \rightarrow 0$ (corresponding to a critical exponent $\gamma_{T}=\infty$ ). For the nearly polarized state we immediately find $\sigma(T, h) \simeq$ $1-\left(\tau / h_{c}\right) \exp \left[-\left(S \tau / h_{c}\right)\left(\left(h_{c}-h\right) / T\right)\right]$ and $\chi_{\|} \simeq\left(S / h_{c}\right)\{1-$ $\left.\left(S \tau / h_{c}\right)(\tau / T) e^{-\left(S \tau / h_{c}\right)\left(\left(h_{c}-h\right) / T\right)}\right\}$.

Let us consider now the regime $\omega_{0} / T \gg 1$. For $h=h_{c}$, (64) gives

$$
\omega_{0}\left(T, h_{c}\right) \simeq \frac{h_{c} T}{S \tau} \ln \left(\frac{S \tau^{2}}{h_{c} T}\right),
$$

and $\chi_{\perp}\left(T, h_{c}\right)$ diverges as $T^{-1} \ln ^{-1}(1 / T)$ when $T \rightarrow 0$. Moreover, around this vertical line for $T \gg\left|h-h_{c}\right| \ln ^{-1}\left(1 /\left|h-h_{c}\right|\right)$, we find

$$
\omega_{0}(T, h) \simeq=\frac{h_{c} T}{S \tau} \ln \left(\frac{S \tau^{2}}{h_{c} T}\right)\left\{1+\frac{S \tau}{h_{c} T} \frac{h-h_{c}}{\ln \left(S \tau^{2} / h_{c} T\right)}\right\},
$$

which signals the same leading behavior of $\omega_{0 c}(T)$ and related thermodynamic quantities in the limit $T \rightarrow 0$, except for small corrections in $h-h_{c}$.

Finally, for $h>h_{c}$ and $T \ll\left(h-h_{c}\right) \ln ^{-1}\left(1 /\left(h-h_{c}\right)\right)$, the frequency gap behaves as

$$
\omega_{0}(T, h) \simeq\left(h-h_{c}\right)\left\{1-\frac{T}{S \tau} \frac{h_{c}}{h-h_{c}} \ln \left(\frac{\tau}{S h_{c}} \frac{h_{c}}{h-h_{c}}\right)\right\},
$$

providing a thermodynamics very similar to the one found for $d<2$ except for logarithmic corrections in $h-h_{c}$ with respect to the $T=0 \mathrm{MF}$ results.

In conclusion, for the two-dimensional CPFM close to $(T=0)-\mathrm{CP}$, we have a qualitative phase diagram which is very similar to the one shown in Figure 2, presenting three regions $C, Q_{1}$, and $Q_{2}$. In this case the sector corresponding to previous $Q_{1}$ is absent and the $\mathrm{V}$-shaped region is delimited by the lines $T_{1,2}(h) \sim\left|h-h_{c}\right| \ln ^{-1}\left(1 /\left|h-h_{c}\right|\right)$ which, in contrast to the ones shown in Figure 2, exhibit small logarithmic corrections.

4.3. $d>2$. For such dimensionalities the CPFM exhibits a critical line ending in the $(T=0)$-CP. To explore the lowtemperature critical properties it is convenient to rewrite (52) 
in terms of $g(T)=h-h_{c}(T) \geq 0$ (for the disordered phase). We will focus on dimensionalities $2<d<4$ (the case $d \geq$ 4 is rather trivial although completely consistent with the general theory of critical phenomena) for which, given the expansions (53), (52) assumes the form

$$
\frac{\omega_{0}}{T} \simeq \frac{g(T)}{T}-\frac{\pi d / 2}{|\sin (\pi d / 2)|}\left(\frac{h_{c}}{S \tau}\right)\left(\frac{T}{\tau}\right)^{(d-2) / 2}\left(\frac{\omega_{0}}{T}\right)^{(d-2) / 2},
$$

where the right-hand side must be positive for stability reasons.

In this paper we will present only explicit results related to the transverse thermodynamics which plays a direct role for our purposes. However, the relevant longitudinal quantities near the polarized state may be simply obtained from the general relations $\sigma=h / h_{c}-\omega_{0} / h_{c}$ and $\chi_{\|}=S(\partial \sigma / \partial h)$.

We start calculating the asymptotic solutions of (69) where the critical region around the $(T=0)-\mathrm{CP}$ in the $(h, T)$-plane is approached in two ways: (i) along horizontal trajectories, as $h \rightarrow h_{c}^{+}(T)$ (with $h_{c}(T) \simeq h_{c}-(d /(d-2)$ ) $\left(h_{c} / S\right)(T / \tau)$ ) at fixed $T$ (isothermal trajectories); and (ii) along vertical trajectories, as $T \rightarrow T_{c}^{+}(h)$ (with $T_{c}(h) \simeq$ $\left.((d-2) / d)\left(S \tau / h_{c}\right)\left(h_{c}-h\right)\right)$ at fixed $h \leq h_{c}$ or $T \rightarrow 0$ for $h>h_{c}$.

We first consider the regime $\omega_{0} / T \ll 1\left(T \xi_{\perp}^{2} \gg 1\right)$. The right-hand side of (69) suggests that two subregimes $\omega_{0} / T \ll$ $\theta$ and $\omega_{0} / T \gg \theta$ should be investigated, with

$$
\theta=\frac{\pi d / 2}{|\sin (\pi d / 2)|}\left(\frac{h_{c}}{S \tau}\right)\left(\frac{T}{\tau}\right)^{(d-2) / 2}\left(\frac{\omega_{0}}{T}\right)^{(d-2) / 2},
$$

where $\omega_{0} / T \simeq \theta$ signals the crossover between them. For isothermal trajectories, in the subregime $\omega_{0} / T \ll \theta$, one finds for $\omega_{0}$ the asymptotic solution

$$
\begin{aligned}
\omega_{0}(T, h) \simeq & \tau\left[\frac{\pi d / 2}{|\sin (\pi d / 2)|} \frac{h_{c}}{S}\right]^{-2 /(d-2)}\left(\frac{T}{\tau}\right)^{-2 /(d-2)} \\
& \times\left(h-h_{c}(T)\right)^{(d-2) / 2}
\end{aligned}
$$

which provides for transverse susceptibility $\chi_{\perp} \sim$ $\left(h-h_{c}(T)\right)^{-\gamma_{h}}$ the nontrivial non-MF critical exponent $\gamma_{h}=2 /(d-2)$. This spherical-model incorrect result is typical of the Tyablikov-like decoupling also for the quantum model at finite temperature $[28,39,40]$.

When $\omega_{0} / T \gg \theta,(69)$ yields simply $\omega_{0} \simeq h-h_{c}(T)$ which corresponds to the MF exponent $\gamma_{h}=1$. The crossover between the two previous regimes $\left(\omega_{0} / T \simeq \theta\right)$ is indicated by the Ginzburg-like line for horizontal trajectories:

$$
\begin{aligned}
h_{\mathrm{Gi}}(T) \simeq & h_{c}(T)+h_{c}\left[\frac{1}{S} \frac{\pi d / 2}{|\sin (\pi d / 2)|}\right]^{2 /(4-d)} \\
& \times\left(\frac{h_{c}}{\tau}\right)^{(d-2) /(4-d)}\left(\frac{T}{\tau}\right)^{2 /(4-d)} .
\end{aligned}
$$

Notice that $h_{\mathrm{Gi}}(T) \rightarrow h_{c}$ as $T \rightarrow 0$; that is, the two lines $h_{c}(T)$ and $h_{\mathrm{Gi}}(T)$ merge at the $(T=0)-\mathrm{CP}$.
For vertical trajectories at fixed $h<h_{c}\left(T_{c}(h) \neq 0\right)$, setting $g(T) \simeq(d /(d-2))\left(h_{c} / S \tau\right)\left(T-T_{c}(h)\right)$ in (69), we easily obtain the asymptotic solutions.

$$
\omega_{0} \simeq \begin{cases}\tau\left[\frac{d-2}{2} \frac{\mid \sin (\pi d / 2)}{\pi d / 2}\right]^{2 /(d-2)}\left(\frac{T-T_{c}(h)}{T_{c}(h)}\right)^{2 /(d-2)}, & \frac{\omega_{0}}{T} \ll \theta, \\ \frac{d}{d-2} \frac{h_{c}}{S}\left(\frac{T_{c}(h)}{\tau}\right)\left(\frac{T-T_{c}(h)}{T_{c}(h)}\right), & \frac{\omega_{0}}{T} \gg \theta .\end{cases}
$$

All the macroscopic quantities of interest can be now determined in the previous regimes for $T \rightarrow T_{c}^{+}(h)$. In particular, for $\chi_{\perp}$, we find the critical exponents $\gamma_{T} \equiv \gamma_{h}=$ $2 /(d-2)$ for $\omega_{0} / T \ll \theta$ and $\gamma_{T}=\gamma_{h}=1$ for $\omega_{0} / T \gg \theta$. The crossover between these two asymptotic sub-regimes for $h<h_{c}$ occurs crossing the conventional Ginzburg-Landau line (with $\omega_{0} / T \simeq \theta$ ):

$$
\begin{aligned}
T_{G i}(h) \simeq & T_{c}(h)+\frac{d-2}{d} \tau\left[\frac{\pi d / 2}{|\sin (\pi d / 2)|}\right]^{2 /(4-d)} \\
& \times\left(\frac{h_{c}}{S \tau}\right)^{(d-2) /(4-d)}\left(\frac{T_{c}(h)}{\tau}\right)^{2 /(4-d)} .
\end{aligned}
$$

Of course, also for vertical trajectories within the region of the $(h, T)$-plane between the critical and Ginzburg lines, with $h<h_{c}$, the TD quantitatively fails.

Let us consider now the behavior of $\omega_{0}$, and hence of $\chi_{\perp}$, along the line $h=h_{c}$, decreasing $T$, which is of most experimental interest in view of the problematics discussed in Section 1.

Since in this case $T_{c}\left(h_{c}\right)=0$, one can immediately see that, to leading order in $T$, a self-consistent solution of (69), under the condition $\omega_{0} / T=O(1)$, is given by

$$
\omega_{0 c}(T) \simeq \frac{d}{d-2}\left(\frac{h_{c}}{S}\right)\left(\frac{T}{\tau}\right) .
$$

This result is strictly connected with the shift exponent $\psi=$ 1 and in drastic contrast with the corresponding relation obtained for the QPFM $[27,39]$, which, due to the presence of quantum fluctuations, shows that $\psi=d / 2$. Equation (75) predicts that $\chi_{\perp} \sim T^{-1}$ as $T \rightarrow 0$ along the vertical line $h=$ $h_{c}$, providing the exponent $\gamma_{T}=\psi=1$, in contrast with the quantum result $\gamma_{T}=\psi=d / 2$ for the QPFM $[28,39]$.

From (69), under condition $h-h_{c} \ll(d /(d-$ 2)) $\left(h_{c} / S\right)(T / \tau)$, we get

$$
\omega_{0}(T, h) \simeq \omega_{0 c}(T)\left\{1+\frac{d-2}{d}\left(\frac{S \tau}{h_{c}}\right) \frac{\left(h-h_{c}\right)}{T}\right\} .
$$

This means that, within the $\mathrm{V}$-shaped region delimited by the critical line for $h<h_{c}$ and the symmetric one $T^{X}(h) \simeq$ $((d-2) / d)\left(S \tau / h_{c}\right)\left(h-h_{c}\right)$ for $h>h_{c}$, the spectrum gap, the transverse susceptibility, and other macroscopic quantities behave essentially as along the line $h=h_{c}$, except for negligible corrections in $h-h_{c}$.

Increasing $h-h_{c}>0$ and crossing the line $T^{X}(h)$, a crossover to the regime $\omega_{0} / T \gg 1\left(T \xi_{\perp}^{2} \ll 1\right)$ takes place 


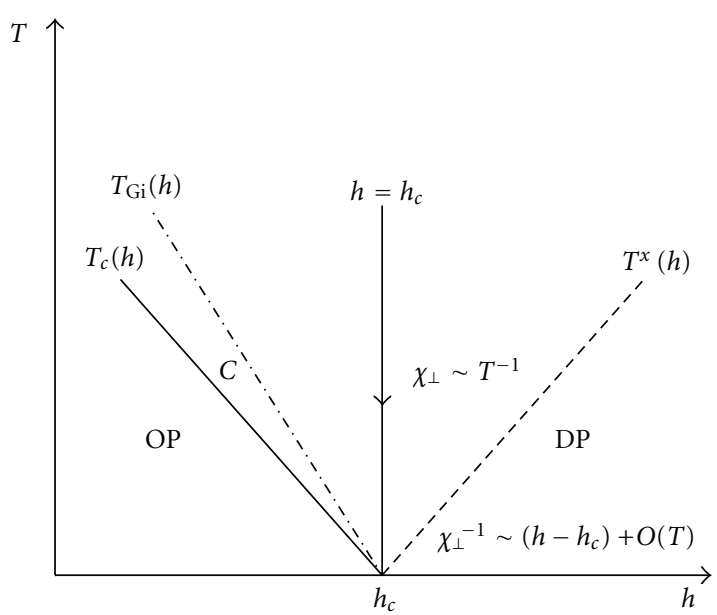

Figure 3: Schematic low-temperature phase diagram and crossovers in the $(h, T)$-plane of the CPFM with $2<d<4$. Here $C$ stands for classical critical behavior; $T_{c}(h), T_{\mathrm{Gi}}(h)$ and $T^{X}(h)$, denote the critical, Ginzburg, and crossover lines; OP and DP indicate the ordered and disordered phases, respectively. The critical line behaves linearly with $h_{c}-h$ and the characteristic quantum-like $\mathrm{V}$-shaped region, with vertex in the $(T=0)-\mathrm{CP}$, is clearly evident.

and one easily finds that, for $T \ll T^{X}(h)$, the appropriate solution of (69) sounds as

$$
\begin{aligned}
\omega_{0}(T, h) \simeq & \left(h-h_{c}\right)+\frac{d}{d-2}\left(\frac{h_{c}}{S \tau}\right) \\
& \times T\left\{1-\frac{\pi d / 2}{|\sin (\pi d / 2)|} \frac{d-2}{d}\left(\frac{h-h_{c}}{\tau}\right)^{(d-2) / 2}\right\} .
\end{aligned}
$$

Therefore, $\chi_{\perp}^{-1}$ (as $\left.\omega_{0}\right)$ behaves essentially as at $T=0\left(\chi_{\perp} \simeq\right.$ $\left.\left(h-h_{c}\right)^{-1}\right)$, with small corrections in $T$.

Summarizing, for $2<d<4$ the low- $T$ global phase diagram has the qualitative structure shown in Figure 3.

A relevant feature of the phase diagram for the CPFM is that it appears qualitatively similar to the one found for the QPFM using different approaches: RG [27] and two-time GF method $[34,39]$. However, for sufficiently low temperatures along the vertical trajectory $h=h_{c}$, the quantitative difference between the behaviors of the transverse susceptibility, $\chi_{\perp} \sim T^{-1}$ of the CPFM and $\chi_{\perp} \sim T^{-d / 2}$, may play a crucial role to distinguish classical and quantum fluctuations in realistic PFM-like systems.

In this framework, it is also worth noting that, as a subproduct of the previous analysis, an identical qualitative global phase diagram for the classical XY model $\left(J^{\|}=0\right)$ in a transverse magnetic field occurs close to its $(T=0)$-CP.

The same low- $T$ V-shaped-like scenario has been recently obtained for the CTIM [22] by means of a Wilsonian RG approach in $d=4-\varepsilon$ dimensions applied to an appropriate Ginzburg-Landau functional representation. This scenario seems to be a common feature of a variety of classical anisotropic magnetic systems which exhibit a $(T=0)-\mathrm{CP}$ as in the quantum counterparts.

\section{Concluding Remarks}

In the present paper we have explored the low-temperature properties of the $d$-dimensional classical planar ferromagnet (CPFM), which exhibits a field-induced zero-temperature critical point, by adopting the two-time Green's function framework in classical statistical mechanics.

It was shown that, close to the $(T=0)-\mathrm{CP}$, the phase diagram and the critical scenario are qualitatively similar to those found for the spin-S QPFM [28] for $d>2$ where a critical line exists. The quantitative differences between the two systems, taking place within the $\mathrm{V}$-shaped region of the phase diagram as the $(T=0)-\mathrm{CP}$ is approached along vertical trajectories, might allow to understand when classical and quantum fluctuations are active. These discrepancies are related to the value of the shift exponent $\psi$ which characterizes the way in which the critical line ends at the $(T=0)$-CP as $T \rightarrow 0$ for classical $(\psi=1)$ and quantum $(\psi=d / 2)$ PFM. In view of our results, we argue that the experimental investigation of the low-temperature criticality of PFM-like systems along and near the line $h=h_{c}$ (quantum critical trajectory for the QPFM) in the $(h, T)$-plane and, in particular, precise measurements of the critical exponents for the correlation function or the susceptibility may provide a signature of the increase of the shift exponent from $\psi=1$ to $\psi=d / 2$ at a certain crossover temperature from the CPFM regime to the quantum one. In particular, when the quantum fluctuations become active, a dimensionality dependence of the shift exponent and related ones should emerge, in contrast with the classical region where $\psi=1$ for any $d>2$. This may be a useful guide to establish where the thermal fluctuations dominate over the quantum ones, and vice versa. Therefore, it should be of experimental interest to estimate the crossover temperature below which the quantum critical fluctuations are expected to govern the physics close to the $(T=0)$-CP. Of course, this requires necessarily an appropriate study of quantum spin models within their V-shaped region.

Useful insights into this problem may be provided by a recent RG analysis [27] for spin-1/2 QPFM with short range interactions, and by two-time GF approaches for spin$1 / 2$ [39] and spin-S [28] QPFM for short- and long-range interactions. In these papers, an unexpected regime with $\chi_{\perp} \sim T^{-1}$ was found in the $\mathrm{V}$-shaped region above a certain temperature $T^{*}$ which, for $2<d<4$, spin-1/2, and short-range interactions at a TD level, reads [39] (using our notations)

$$
T^{*} \simeq \tau\left[\frac{1}{\pi}|\sin (\pi d / 2)|\left(F_{d / 2}(0)\right)^{(4-d) / 2}\right]^{4 /(d-2)^{2}}\left(\frac{\tau}{d h_{c}}\right)^{2 /(d-2)},
$$

where $F_{d / 2}(0)$ is the value at $y=0$ of the function $F_{d / 2}(y)=$ $\int_{0}^{\infty} d x\left(x^{(d / 2)-1} /\left(e^{x+y}-1\right)\right)$. Below $T^{*}$, the quantum behavior $\chi_{\perp} \sim T^{-d / 2}$ takes place, as expected from the paradigmatic quantum critical scenario [1].

The present study for the CPFM clarified the physical meaning of $T^{*}$. Our study corroborated the idea that, decreasing $T$ along the quantum critical trajectory, $T^{*}$ provides an estimate of the temperature which signals 
a crossover between the classical thermally activated regime, with $\chi_{\perp} \sim T^{-1}(\psi=1)$, and the quantum one, with $\chi_{\perp} \sim$ $T^{-d / 2}(\psi=d / 2)$. This feature agrees with the RG predictions for the same quantum spin model near and below four dimensions [27].

It is worth mentioning that within our many-body framework one can also extract the basic quantum-like dynamics by variation of dimensionality. In particular, relevant information can be easily obtained from the scaling structure of the transverse dynamic susceptibility $\chi_{\perp}(\mathbf{k}, \omega)=$ $-G_{\perp}(\mathbf{k}, \omega)$ for small values of the arguments. Indeed, from (33)-(34) close to the ( $T=0)$-CP we find

$$
\chi_{\perp}(\mathbf{k}, \omega) \simeq 2 S \xi_{\perp}^{2}\left\{\left[S J^{\perp}\left(k \xi_{\perp}\right)^{2}+1\right]-\omega \xi_{\perp}^{2}\right\}^{-1}
$$

where $\xi_{\perp} \simeq\left(S J^{\perp}\right)^{1 / 2}\left(h-h_{c}\right)^{-1 / 2} \propto \chi_{\perp}^{1 / 2}$ defines the transverse correlation length at zero temperature for $h \geq h_{c}$. Then, comparing (79) with the general dynamic scaling relation $\chi(\mathbf{k}, \omega) \simeq \xi^{2-\eta} W\left(k \xi, \omega \xi^{z}\right)$ we immediately get $\eta=0$ and $z=2$ for the Fisher $\eta$ and dynamic $z$ critical exponents, and $W(x, y)=2 S\left[S J^{\perp}\left(x^{2}+1\right)-y\right]^{-1}$.

In conclusion, our results are in agreement with the statements made in [21] for the CTIM chain and in [28] for $d>1$. Our analysis suggests that reliable measurements of the shift exponent (or related ones) close to the QCP of magnetic systems with PFM symmetry may provide a signature of the presence of quantum critical fluctuations. We believe also that this feature is rather general and not limited to TIM-like and PFM-like systems.

\section{Appendices}

\section{A. An Outline of the Two-Time Green's Function Framework in Classical Statistical Mechanics}

In this section, for utility of the reader, we briefly review the basic ingredients of the two-time retarded $(\nu=r)$ and advanced $(\nu=a)$ GF's framework in classical statistical mechanics in a form strictly parallel to the quantum counterpart [40-44]. For two arbitrary dynamical variables $A$ and $B$, they are defined as [29]

$$
\begin{aligned}
G_{A B}^{(v)}\left(t, t^{\prime}\right) & =\theta_{\nu}\left(t-t^{\prime}\right)\left\langle\left\{A(t), B\left(t^{\prime}\right)\right\}\right\rangle \\
& \equiv\left\langle\left\langle A(t) ; B\left(t^{\prime}\right)\right\rangle\right\rangle_{\nu}, \quad(\nu=r, a),
\end{aligned}
$$

where $\theta_{r}\left(t-t^{\prime}\right)=\theta\left(t-t^{\prime}\right), \theta_{a}\left(t-t^{\prime}\right)=-\theta\left(t^{\prime}-t\right), \theta(x)$ is the usual step function, $\langle\cdots\rangle$ denotes an equilibrium ensemble average, and $\{A, B\}$ is the Poisson bracket of $A$ and $B$.

In (A.1), the dynamical variables $A$ and $B$ depend on time via the conjugate canonical coordinates $(q(t), p(t)) \equiv$ $\left(q_{1}(t), \ldots, q_{\mathcal{N}}(t) ; p_{1}(t), \ldots, p_{\mathcal{N}}(t)\right), \quad(\mathcal{N}$ is the number of degrees of freedom of the classical system under study), $X(t)=e^{i L t} X(0)$ with $X=A, B, q, p, L=i\{\mathscr{H}, \ldots\}$ is the Liouville operator, and $\mathscr{H}$ is the Hamiltonian of the system and $X(0) \equiv X(q(0), p(0))$ at the initial time $t=0$. Of course, the time evolution of the generic dynamical variable $X(t)$ is governed by the well-known Liouville equation of motion (EM):

$$
\frac{d X(t)}{d t}=\{X(t), \mathscr{H}\}
$$

One can easily prove that the two-time GFs (A.1) depends on times $t, t^{\prime}$ only through the difference $t-t^{\prime}$, that is

$$
G_{A B}^{(v)}\left(t-t^{\prime}\right)=\left\langle\left\langle A\left(t-t^{\prime}\right) ; B\right\rangle\right\rangle_{\nu}=\left\langle\left\langle A ; B\left(t^{\prime}-t\right)\right\rangle\right\rangle_{\nu},
$$

and the two-time correlation function $F_{A B}\left(t, t^{\prime}\right)=F_{A B}(t-$ $\left.t^{\prime}\right)=\left\langle A(t) B\left(t^{\prime}\right)\right\rangle=\langle A(\tau) B\rangle=\langle A B(-\tau)\rangle$, with $\tau=t-t^{\prime}$, is related to the classical GFs (A.3) by the following relation [29]:

$$
G_{A B}^{(\nu)}(\tau)=\beta \theta_{\nu}(\tau) \frac{d}{d \tau}\langle A(\tau) B\rangle=\beta \theta_{\nu}(\tau)\langle\{A(\tau), \mathscr{H}\} B\rangle,
$$

where $\beta=\left(K_{B} T\right)^{-1}, T$ is the temperature, and $K_{B}$ is the Boltzmann constant (we assume $K_{B}=1$ ). In particular, we have also

$$
\langle\{A(\tau), B\}\rangle=\beta \frac{d}{d \tau}\langle A(\tau) B\rangle=\beta\langle\{A(\tau), \mathscr{H}\} B\rangle .
$$

For $G_{A B}^{(v)}(\tau)$ and $F_{A B}(\tau)$ one can introduce the Fourier transforms:

$$
\begin{aligned}
& G_{A B}^{(v)}(\tau)=\int_{-\infty}^{+\infty} \frac{d \omega}{2 \pi} G_{A B}^{(v)}(\omega) e^{-i \omega \tau} \\
& F_{A B}(\tau)=\int_{-\infty}^{+\infty} \frac{d \omega}{2 \pi} F_{A B}(\omega) e^{-i \omega \tau}
\end{aligned}
$$

where $G_{A B}^{(\nu)}(\omega)=\langle\langle A(\tau) ; B\rangle\rangle_{\nu, \omega}$ and $F_{A B}(\omega)=\langle A(\tau) B\rangle_{\omega}$ are called the $\nu$-GF of $A$ and $B$ in the $\omega$-representation and the classical spectral intensity of the time-dependent correlation function $F_{A B}(\tau)$, respectively, with $f(\omega)=\int_{-\infty}^{+\infty} d \tau e^{i \omega \tau} f(\tau)$. Then, using (A.4) and the integral representations

$$
\begin{gathered}
\theta(\tau)=i \int_{-\infty}^{+\infty} \frac{d x}{2 \pi} \frac{e^{-i x \tau}}{x+i \varepsilon}, \quad \varepsilon \longrightarrow 0^{+} \\
\delta(x)=\int_{-\infty}^{+\infty} \frac{d \tau}{2 \pi} e^{i x \tau},
\end{gathered}
$$

for the step function and the Dirac $\delta$-function, $G_{A B}^{(\nu)}(\omega)$ can be expressed in terms of the corresponding spectral intensity as

$$
G_{A B}^{(\nu)}(\omega)=\int_{-\infty}^{+\infty} \frac{d \omega^{\prime}}{2 \pi} \frac{\beta \omega^{\prime} F_{A B}\left(\omega^{\prime}\right)}{\omega-\omega^{\prime}+(-1)^{\nu} i \varepsilon}, \quad \varepsilon \longrightarrow 0^{+},
$$

where the symbol $(-1)^{\nu}$ means +1 if $v=r$ and -1 if $v=a$. It is interesting to compare (A.8) with the quantum corresponding expression for two operators $A$ and $B$ [40-44]:

$$
G_{A B}^{(\nu)}(\omega)=\int_{-\infty}^{+\infty} \frac{d \omega^{\prime}}{2 \pi} \frac{\left(1+\eta e^{-\beta \hbar \omega^{\prime}}\right) F_{A B}\left(\omega^{\prime}\right)}{\omega-\omega^{\prime}+(-1)^{\nu} i \varepsilon}, \quad \varepsilon \longrightarrow 0^{+},
$$

where $\eta=-1$ and $\eta=+1$ by definition of quantum twotime GFs with commutator or anticommutator, respectively, 
and $\hbar$ is the reduced Planck constant. Notice that formally, as expected for internal consistency, the function $C(\omega)=\beta \omega$ or $Q_{\eta}(\omega)=\left(1+\eta e^{-\beta \hbar \omega}\right) / \hbar$ characterizes the classical or quantum nature of the problem under study, respectively.

In analogy with the quantum case [45-47], we now introduce the time-dependent classical spectral density (CSD) for $A$ and $B[31,37,48,49]$ :

$$
\Lambda_{A B}(\tau)=i\langle\{A(\tau), B\}\rangle,
$$

with the Fourier transform:

$$
\Lambda_{A B}(\omega)=i\langle\{A(\tau), B\}\rangle_{\omega}=\int_{-\infty}^{+\infty} d \tau e^{i \omega \tau} \Lambda_{A B}(\tau)=\beta \omega F_{A B}(\omega) .
$$

Hence, from (A.8), one immediately obtains the spectral representation:

$$
G_{A B}^{(\nu)}(\omega)=\int_{-\infty}^{+\infty} \frac{d \omega^{\prime}}{2 \pi} \frac{\Lambda_{A B}\left(\omega^{\prime}\right)}{\omega-\omega^{\prime}+(-1)^{v} i \varepsilon}, \quad \varepsilon \longrightarrow 0^{+},
$$

for the two-time GFs (A.1) in terms of the corresponding $\operatorname{CSD} \Lambda_{A B}(\omega)$ in the $\omega$-representation. Also the dynamical correlation function $\langle A(\tau) B\rangle$ can be easily expressed in terms of $\Lambda_{A B}(\omega)$. From (A.5), (A.10), and (A.11), we obtain indeed (classical spectral theorem)

$$
\langle A(\tau) B\rangle \equiv\langle B A(\tau)\rangle=\int_{-\infty}^{+\infty} \frac{d \omega}{2 \pi} \frac{\Lambda_{A B}(\omega)}{\beta \omega} e^{-i \omega \tau} .
$$

From (A.10)-(A.13) some formally exact results can be easily obtained. First, (A.10) and (A.11) yield

$$
\int_{-\infty}^{+\infty} \frac{d \omega}{2 \pi} \Lambda_{A B}(\omega)=i\langle\{A, B\}\rangle .
$$

Besides, from (A.13), it follows

$$
\int_{-\infty}^{+\infty} \frac{d \omega}{2 \pi} \frac{\Lambda_{A B}(\omega)}{\beta \omega}=\langle A B\rangle .
$$

The relations (A.14) and (A.15) constitute useful examples of the so-called sum rules of the CSD, $\Lambda_{A B}(\omega)$, which have great relevance for physical consistency of practical calculations and approximations. Combining now (A.12) and (A.14), one can easily obtain another general result which plays an important role for calculation of the GFs. As $\omega \rightarrow \infty$ we have indeed [33]

$$
G_{A B}^{(\nu)}(\omega)=\left\{\begin{array}{cl}
\frac{i\langle\{A, B\}\rangle}{\omega} \sim \omega^{-1}, & \text { if }\langle\{A, B\}\rangle \neq 0, \\
\sim \omega^{-\alpha}(\alpha \geq 2), & \text { if }\langle\{A, B\}\rangle=0,
\end{array}\right.
$$

which provide a relevant boundary condition for the $\nu$-GFs.

Let us come back now to the relations (A.12) for classical retarded and advanced GFs in the $\omega$-representation. As in the quantum counterpart [40-42], one can prove that $G_{A B}^{(r)}(\omega)$ and $G_{A B}^{(a)}(\omega)$, analytically continued in the $\omega$-complex plane, are analytical functions in the upper and lower halfplane, respectively. Then, combining these two analytical functions, one can construct a single function $G_{A B}(\omega)=$ $\int_{-\infty}^{+\infty} d t e^{i \omega t} G_{A B}(t)$ of complex $\omega$ such that

$$
G_{A B}(\omega)= \begin{cases}G_{A B}^{(r)}(\omega), & \operatorname{Im} \omega>0 \\ G_{A B}^{(a)}(\omega), & \operatorname{Im} \omega<0\end{cases}
$$

Hence, (A.12) provides for $G_{A B}(\omega)$ the spectral representation:

$$
G_{A B}(\omega)=\int_{-\infty}^{+\infty} \frac{d \omega^{\prime}}{2 \pi} \frac{\Lambda_{A B}\left(\omega^{\prime}\right)}{\omega-\omega^{\prime}} .
$$

This function is analytical in the whole complex $\omega$-plane with a cut along the real axis where singularities for $G_{A B}(\omega)$ may occur. It is worth noting that, in terms of $\Lambda_{A B}(\omega)$, no formal differences exist for the spectral representations of $G_{A B}^{(\nu)}(\omega)$ and $G_{A B}(\omega)$ in the classical and quantum context. Hence, all the developments already known in the quantum framework remain formally valid for the classical one. In particular, one has the important exact relation

$$
\Lambda_{A B}(\omega)=i\left[G_{A B}(\omega+i \varepsilon)-G_{A B}(\omega-i \varepsilon)\right],
$$

which expresses the CSD in terms of the related two-time GFs in the $\omega$-representation. This allows us to state also that the cut for $G_{A B}(\omega)$ along the real axis in $\omega$-complex plane is determined by (A.19) and its singularities are the points of the real axis where the condition $\Lambda_{A B}(\omega) \neq 0$ is satisfied. For the spectral intensity of classical systems, (A.11) and (A.19) yield

$$
F_{A B}(\omega)=\langle A(\tau) B\rangle_{\omega}=i \frac{G_{A B}(\omega+i \varepsilon)-G_{A B}(\omega-i \varepsilon)}{\beta \omega} .
$$

Of course, other known quantum relations are formally valid for classical many-body theory. Besides, when $\Lambda_{A B}(\omega)$ is real, the classical Kramers-Kronig relations (classical dispersion relations) between the real and imaginary parts of $G_{A B}^{(\nu)}(\omega)$ are true:

$$
\operatorname{Re} G_{A B}^{(\nu)}(\omega)=\frac{(-1)^{(\nu)}}{\pi} \wp \int_{-\infty}^{+\infty} d \omega^{\prime} \frac{\operatorname{Im} G_{A B}^{(\nu)}\left(\omega^{\prime}\right)}{\omega^{\prime}-\omega},
$$

where the symbol $\wp$ denotes the main part of the integral. We have also

$$
\Lambda_{A B}(\omega)=-2(-i)^{\nu} \operatorname{Im} G_{A B}^{(\nu)}(\omega),
$$

and, in particular,

$$
\Lambda_{A B}(\omega)=-2 \operatorname{Im} G_{A B}^{(r)}(\omega) .
$$

Differentiating (A.3) with respect to $\tau=t-t^{\prime}$, with $\mathrm{EM}$ (A.2) for dynamical variables and $d \theta_{\nu}(\tau) / d \tau=\delta(\tau)$, yields

$$
\frac{d}{d \tau}\langle\langle A(\tau) ; B\rangle\rangle_{\nu}=\delta(\tau)\langle\{A, B\}\rangle+\langle\langle\{A(\tau), \mathcal{H}\} ; B\rangle\rangle_{\nu},
$$

which is the basic EM for the GF $\langle\langle A(\tau) ; B\rangle\rangle_{\nu}$. This, however, is not a closed differential equation since in the right-hand side of (A.24) a new higher-order $\nu$-GF occurs involving Poisson brackets of a greater number of dynamical variables. 
Then, one needs to consider a new EM for the two-time $\nu$-GF $\langle\langle\{A(\tau), \mathscr{H}\} ; B\rangle\rangle_{\nu}$. The $\tau$-derivative of this function provides an additional equation, formally identical to (A.24) with $A(\tau)$ replaced by $\{A(\tau), \mathscr{H}\}$, the right-hand side of which contains the new $\nu$-GF $\langle\langle\{\{A(\tau), \mathscr{H}\}, \mathscr{H}\} ; B\rangle\rangle_{\nu}$. By iteration of this procedure, we obtain the infinite chain of coupled EMs for GFs of increasing order:

$$
\begin{aligned}
\frac{d}{d \tau}\left\langle\left\langle\mathcal{L}_{\mathscr{H}}^{m} A(\tau) ; B\right\rangle\right\rangle_{\nu}= & \delta(\tau)\left\langle\left\{\mathcal{L}_{\mathscr{H}}^{m} A, B\right\}\right\rangle \\
& +\left\langle\left\langle\mathcal{L}_{\mathscr{H}}^{m+1} A(\tau) ; B\right\rangle\right\rangle_{\nu} \quad(m=0,1,2, \ldots) .
\end{aligned}
$$

Here $\mathcal{L}_{\mathscr{H}}=i L=\{\ldots, \mathscr{H}\}$ and $\mathcal{L}_{\mathscr{H}}^{m} A$ means $\mathcal{L}_{\mathscr{H}}^{0} A=A$, $\mathcal{L}_{\mathscr{H}}^{1} A=\{A, \mathscr{H}\}, \mathcal{L}_{\mathscr{H}}^{2} A=\{\{A, \mathscr{H}\}, \mathscr{H}\}$, and so on. Notice that the chain of EMs (A.25) is formally the same for different types of GFs and hence one can eliminate the index $v$ when the physical context is clear.

In the practical calculations it is more convenient to work in the $\omega$-Fourier space. With $i \int_{-\infty}^{+\infty} d \tau e^{i \omega \tau}(d f(\tau) / d \tau)=$ $\omega f(\omega)$, the chain of equations, in the $\omega$-representation, assumes the following form:

$$
\begin{aligned}
\omega\left\langle\left\langle\mathcal{L}_{\mathscr{H}}^{m} A(\tau) ; B\right\rangle\right\rangle_{\nu, \omega}= & i\left\langle\left\{\mathcal{L}_{\mathscr{H}}^{m} A, B\right\}\right\rangle \\
& +i\left\langle\left\langle\mathcal{L}_{\mathcal{H}}^{m+1} A(\tau) ; B\right\rangle\right\rangle_{\nu, \omega} \quad(m=0,1,2, \ldots),
\end{aligned}
$$

which has to be solved with appropriate boundary conditions (A.17). Since an exact solution is, of course, impossible, in practical calculations one must resort to decoupling procedures, and hence to approximate methods, to reduce the infinite chain of coupled equations to a finite closed one. Unfortunately, systematic and controllable decouplings are not easy to find and one must check for the reliability of a given approximation for each specific problem, by comparing the results with experiments, simulations, or other types of approaches.

\section{B. Callen-Like Approach for Magnetization: A Classical Moment Problem}

In this appendix we present an instructive method to solve the differential equation (25) for $\Omega(a)$ via a classical moment problem. The differential equation is the following:

$$
\Omega^{\prime \prime}(a)+2\left(\frac{1}{\Phi}+a\right)^{-1} \Omega^{\prime}(a)-S^{2} \Omega(a)=0,
$$

for which the initial condition $\Omega(0)=1$ is valid by definition. This is, of course, insufficient to find the physical solution of (25) and one should add a supplementary condition to be searched properly. Unfortunately, there is not classical analogue of the operatorial identity $\Pi_{p=-S}^{S}\left(S^{z}-p\right)=0$ which is the key ingredient of the Callen approach for the quantum $\mathrm{HM}$ [35]. In the following, we will show that, at our level of approximation, the additional condition

$$
\Omega(a)=\int_{-S}^{S} d S^{z} f\left(S^{z}\right) e^{a S^{z}},
$$

which follows formally from the definition of the canonical ensemble average of the dynamical variable $e^{a S^{z}}$, combined with $\Omega(0)=1$, allows to determine $\Omega(a)$ as a classical moment problem [50-53]. In view of the structure of the differential equation (25) for $\Omega(a)$, it is convenient to define

$$
f\left(S^{z}\right)=g\left(S^{z}\right) e^{S^{z} / \Phi}
$$

so that, we can write

$$
\begin{aligned}
\Omega(a) & =\int_{-S}^{S} d S^{z} g\left(S^{z}\right) e^{(1 / \Phi+a) S^{z}} \\
& =S \int_{-1}^{1} d x g(S x) e^{S(1 / \Phi+a) x} .
\end{aligned}
$$

Replacing this expression and its derivatives in (25), we have for $g\left(S^{z}\right)$ the following:

$$
\int_{-1}^{1} d x\left(y x^{2}+2 x-y\right) g(S x) e^{y x}=0
$$

where $y=S(1 / \Phi+a)$. Then, with $e^{y x}=\sum_{n=0}^{\infty}\left(y^{n} / n !\right) x^{n},($ B.5 $)$ provides

$$
\sum_{n=0}^{\infty} \frac{y^{n}}{n !}\left[y\left(I_{n+2}-I_{n}\right)+2 I_{n+1}\right]=0,
$$

with

$$
I_{k}=\int_{-1}^{1} d x g(S x) x^{k}
$$

It is now simple to show that the moments (B.7) of $g(S x) \equiv$ $g(x)$ are determined by the recursion relations:

$$
I_{1}=0, \ldots, I_{n+1}=\frac{n}{n+2} I_{n-1}, \quad n=1,2, \ldots
$$

These provide

$$
I_{k}= \begin{cases}\frac{I_{0}}{k+1}, & k=2 n, \\ 0, & k=2 n+1, n \geq 0,\end{cases}
$$

where, at this stage, the moment $I_{0}$ is unknown. From (B.9), it is immediate to see that the solution of the moment problem for $g(S x)$ is given by $g(S x)=I_{0} / 2$.

However, for the univocal determination of $\Omega(a)$ it is not necessary to have the explicit form of $g(S x)=g\left(S^{z}\right)$ but rather its moments (B.9). From the integral representation (B.4), we have indeed

$$
\begin{aligned}
\Omega(a) & =S \sum_{k=0}^{\infty} \frac{y^{k}}{k !} \int_{-1}^{1} d x g(S x) x^{k} \\
& =\frac{S I_{0}}{y} \sum_{n=0}^{\infty} \frac{y^{2 n+1}}{(2 n+1) !}=\frac{S I_{0}}{y} \sinh y
\end{aligned}
$$

or, explicitly,

$$
\Omega(a)=S I_{0} \frac{\sinh [S(1 / \Phi+a)]}{S(1 / \Phi+a)} .
$$


Finally, the boundary condition $\Omega(0)=1$ yields $I_{0}=(1 / \Phi)$ $(1 / \sinh (S / \Phi))$ so that we obtain the following solution:

$$
\Omega(a)=\frac{1 / \Phi}{1 / \Phi+a} \frac{\sinh [S(1 / \Phi+a)]}{\sinh (S / \Phi)} .
$$

This is the central result of this appendix which constitutes the classical analogue [34] of the quantum Callen formula [35]. It provides the required expression for $m$, which is valid for any $d, T$, and $h$.

\section{Acknowledgments}

A. Cavallo acknowledges the MIUR (Italian Ministry of Research) for financial support within the program "Incentivazione alla mobilità di studiosi stranieri e italiani residenti all'estero."

\section{References}

[1] S. Sachdev, Quantum Phase Transitions, Cambridge University Press, Cambridge, UK, 1999.

[2] G. R. Stewart, "Non-fermi-liquid behavior in d-and f-electron metals," Reviews of Modern Physics, vol. 73, no. 4, pp. 797-855, 2001.

[3] H. V. Löhneysen, A. Rosch, M. Vojta, and P. Wölfle, "Fermiliquid instabilities at magnetic quantum phase transitions," Reviews of Modern Physics, vol. 79, no. 3, pp. 1015-1075, 2007.

[4] P. Gegenwart, Q. Si, and F. Steglich, "Quantum criticality in heavy-fermion metals," Nature Physics, vol. 4, no. 3, pp. 186197, 2008.

[5] S. Roy and A. M. S. Tremblay, "Scaling and commensurateincommensurate crossover for the $\mathrm{d}=2, \mathrm{z}=2$ quantum critical point of itinerant antiferromagnets," Europhysics Letters, vol. 84, no. 3, Article ID 37013, 6 pages, 2008.

[6] J. Kroha, M. Klein, A. Nuber, F. Reinert, O. Stockert, and H. V. Löhneysen, "High-temperature signatures of quantum criticality in heavy-fermion systems," Journal of Physics Condensed Matter, vol. 22, no. 16, Article ID 164203, 2010.

[7] J. A. Hertz, "Quantum critical phenomena," Physical Review B, vol. 14, no. 3, pp. 1165-1184, 1976.

[8] A. J. Millis, "Effect of a nonzero temperature on quantum critical points in itinerant fermion systems," Physical Review B, vol. 48, no. 10, pp. 7183-7196, 1993.

[9] T. Moriya, Spin Fluctuations in Itinerant Electron Magnetism, Springer, Berlin, Germany, 1985.

[10] T. Moriya and T. Takimoto, "Anomalous properties around magnetic instability in heavy electron systems," Journal of the Physical Society of Japan, vol. 64, no. 3, pp. 960-969, 1995.

[11] S. Takashima, M. Nohara, H. Ueda et al., "Robustness of nonFermi-liquid behavior near the ferromagnetic critical point in clean ZrZn2," Journal of the Physical Society of Japan, vol. 76, no. 4, Article ID 043704, 2007.

[12] R. A. Borzi, S. A. Grigera, J. Farrell et al., "Formation of a nematic fluid at high fields in Sr3Ru 2O7," Science, vol. 315, no. 5809, pp. 214-217, 2007.

[13] T. Misawa, Y. Yamaji, and M. Imada, "Spin fluctuation theory for quantum tricritical point arising in proximity to first-order phase transitions: applications to heavy-fermion systems, YbRh2Si2, CeRu2Si2, and $\beta$-YbAIB4," Journal of the Physical Society of Japan, vol. 78, no. 8, Article ID 084707, 2009.

[14] P. Pfeuty, "The one-dimensional Ising model with a transverse field," Annals of Physics, vol. 57, no. 1, pp. 79-90, 1970.
[15] P. Pfeuty, "The quantum-classical crossover critical behaviour of the Ising model in a transverse field," Journal of Physics $C$, vol. 9, no. 21, pp. 3993-4001, 1976.

[16] B. K. Chakrabarty, A. Dutta, and P. Sen, Quantum Ising Phases and Transitions in Transverse Ising Model, Springer, Berlin, Germany, 1986.

[17] A. K. Chandra, J. I. Inoue, and B. K. Chakrabarti, "Quantum phase transition in a disordered long-range transverse Ising antiferromagnet," Physical Review E, vol. 81, no. 2, Article ID 021101, 2010.

[18] A. Dutta, U. Divakaran, D. Sen, B. K. Chakrabarty, T. F. Rosenbaum, and G. Aeppli, "Transverse field spin models: from Statistical Physics to Quantum Information," Statistical Mechanics. In press. http://arxiv.org/abs/1012.0653.

[19] P. Prelovsek and I. Sega, "Numerical simulation of the classical Ising model in a transverse field statical properties," Journal of Physics C, vol. 11, no. 10, pp. 2103-2113, 1978.

[20] P. Prelovek and I. Sega, "Molecular-dynamics study of the classical Ising model in a transverse field," Physical Review B, vol. 17, no. 11, pp. 4416-4425, 1978.

[21] A. Cuccoli, A. Taiti, R. Vaia, and P. Verrucchi, "Extracting signatures of quantum criticality in the finite-temperature behavior of many-body systems," Physical Review B, vol. 76, no. 6, Article ID 064405, 2007.

[22] A. Caramico D’Auria, L. De Cesare, M. T. Mercaldo, and I. Rabuffo, "Quantum-like criticality for a classical transverse Ising model in $4-\varepsilon$ dimensions," European Physical Journal B, vol. 77, no. 3, pp. 419-427, 2010.

[23] T. Matsubara and H. Matsuda, "A lattice model of liquid Helium, I," Progress of Theoretical Physics, vol. 16, p. 569, 1956.

[24] T. Matsubara and H. Matsuda, "A lattice model of liquid helium, II," Progress of Theoretical Physics, vol. 17, p. 19, 1957.

[25] M. E. Fisher, "The theory of equilibrium critical phenomena," Reports on Progress in Physics, vol. 30, no. 2, article 306, pp. 615-730, 1967.

[26] D. K. Dacol, "Low-temperature critical phenomena in the spin-1/2 planar ferromagnet," Journal of Low Temperature Physics, vol. 41, no. 3-4, pp. 349-373, 1980.

[27] M. T. Mercaldo, A. Caramico D'Auria, L. De Cesare, and I. Rabuffo, "Low-temperature critical properties and crossovers of a spin-1/2 planar ferromagnet in 4- $\varepsilon$ dimensions," Physical Review B, vol. 77, no. 18, Article ID 184424, 2008.

[28] L. S. Campana, L. De Cesare, U. Esposito, M. T. Mercaldo, and I. Rabuffo, "Field-induced quantum critical point in planar Heisenberg ferromagnets with long-range interactions: twotime Green's function framework," Physical Review B, vol. 82, no. 2, Article ID 024409, 2010.

[29] N. N. Bogoliubov and B. I. Sadovnikov, “Green's functions and distribution functions in the statistical mechanics of classical systems," Journal of Experimental and Theoretical Physics, vol. 16, p. 482, 1963.

[30] L. S. Campana, A. C. D’Auria, M. D’Ambrosio, U. Esposito, L. De Cesare, and G. Kamieniarz, "Spectral-density method for classical systems: heisenberg ferromagnet," Physical Review B, vol. 30, no. 5, pp. 2769-2775, 1984.

[31] A. Cavallo, F. Cosenza, and L. De Cesare, "Classical Heisenberg ferromagnetic chain with long-range interactions: a spectral density approach," Physical Review B, vol. 66, no. 17, Article ID 174439, 2002.

[32] A. Cavallo, F. Cosenza, and L. De Cesare, "Thermodynamic properties of a classical d-dimensional spin-S Heisenberg ferromagnet with long-range interactions via the spectral density method," Physica A, vol. 332, no. 1-4, pp. 301-317, 2004. 
[33] A. Cavallo, F. Cosenza, and L. De Cesare, "The classical spectral density method at work: the heisenberg ferromagnet," in New Developments in Ferromagnetism Research, V. N. Murray, Ed., Nova Science Publishers, New York, NY, USA, 2006.

[34] L. S. Campana, A. Cavallo, L. De Cesare, U. Esposito, and A. Naddeo, "Callen-like method for the classical Heisenberg ferromagnet," Physica A, vol. 391, no. 4, pp. 1087-1096, 2012.

[35] H. B. Callen, "Green function theory of ferromagnetism," Physical Review, vol. 130, no. 3, pp. 890-898, 1963.

[36] S. K. Ma, Modern Theory of Critical Phenomena, Benjamin, London, UK, 1976.

[37] L. S. Campana, A. C. D’Auria, M. D’Ambrosio, U. Esposito, L. De Cesare, and G. Kamieniarz, "Spectral-density method for classical systems: heisenberg ferromagnet," Physical Review B, vol. 30, no. 5, pp. 2769-2775, 1984.

[38] N. D. Mermin and H. Wagner, "Absence of ferromagnetism or antiferromagnetism in one- or two-dimensional isotropic Heisenberg models," Physical Review Letters, vol. 17, no. 22, pp. 1133-1136, 1966.

[39] L. S. Campana, L. De Cesare, U. Esposito, and M. T. Mercaldo, “Two-time Green's function treatment of field-induced quantum criticality of a d-dimensional easy-plane ferromagnet with longitudinal uniform interactions," Physica A, vol. 388, no. 8, pp. 1446-1462, 2009.

[40] S. V. Tyablikov, Methods in the Quantum Theory of Magnetism, Plenum Press, New York, NY, USA, 1967.

[41] D. N. Zubarev, "2-Time green functions in statistical physics," Uspekhi Fizicheskikh Nauk, vol. 71, no. 1, pp. 71-116, 1960.

[42] D. N. Zubarev, "Double-time green functions in statistical physics," Soviet Physics Uspekhi, vol. 3, no. 3, p. 320, 1960.

[43] N. Majlis, The Quantum Theory of Magnetism, World Scientific, Singapore, 2000.

[44] D. N. Zubarev, Nonequilibrium Statistical Thermodynamics, Consultants Bureau, New York, NY, USA, 1974.

[45] O. K. Kalashnikov and E. S. Fradkin, ZhurnalEksperimentalnoi i Teoreticheskoi Fiziki, vol. 55, p. 607, 1968.

[46] O. K. Kalashnikov and E. S. Fradkin, "The method of spectral densities in quantum statistical mechanics," Journal of Experimental and Theoretical Physics, vol. 28, p. 317, 1969.

[47] O. K. Kalashnikov and E. S. Fradkin, "Spectral density method applied to systems showing phase transitions," Physica Status Solidi B, vol. 59, no. 1, pp. 9-46, 1973.

[48] A. C. D’Auria, L. De Cesare, and U. Esposito, "Spectral density method in classical statistical mechanics," Physics Letters A, vol. 85 , no. 4, pp. 197-200, 1981.

[49] L. S. Campana, A. C. D’Auria, M. D’Ambrosio, L. De Cesare, and U. Esposito, "A proposal to include damping effects in the spectral density approach," Journal of Physics C, vol. 16, no. 17, pp. L549-L553, 1983.

[50] N. I. Akhiezer, The Classical Moment Problem and Some Related Questions in Analysis, Hafner Publishing, New York, NY, USA, 1965.

[51] T. M. Pham Ngoc, "A statistical minimax approach to the Hausdorff moment problem," Inverse Problems, vol. 24, no. 4, Article ID 045018, 1996.

[52] G. D. Lin, "On the moment problems," Statistics \& Probability Letters, vol. 35, no. 1, pp. 85-90, 1997.

[53] R. Mnatsakanov, "Hausdorff moment problem: reconstruction of distributions," Statistics \& Probability Letters, vol. 78, no. 12, pp. 1612-1618, 2008. 

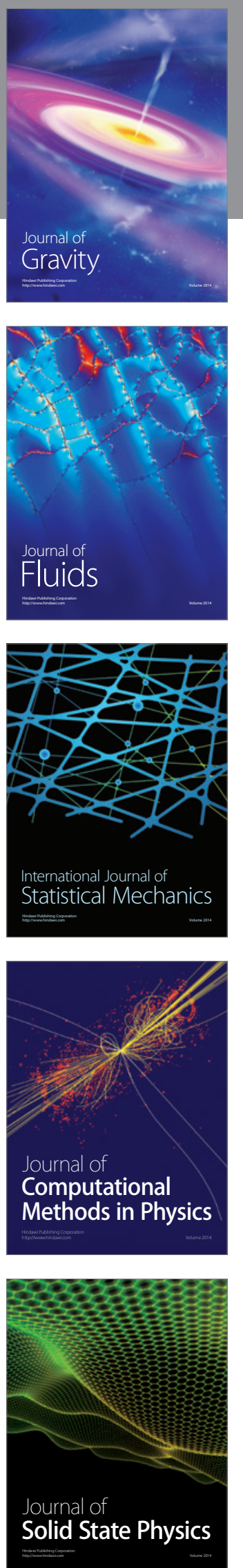

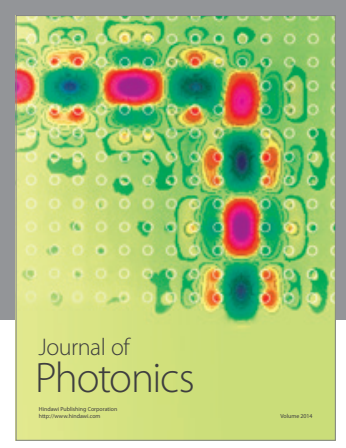

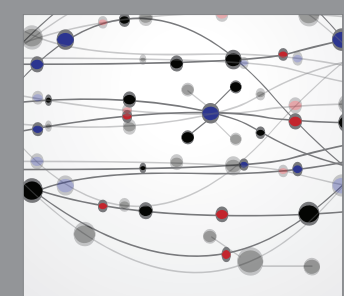

The Scientific World Journal
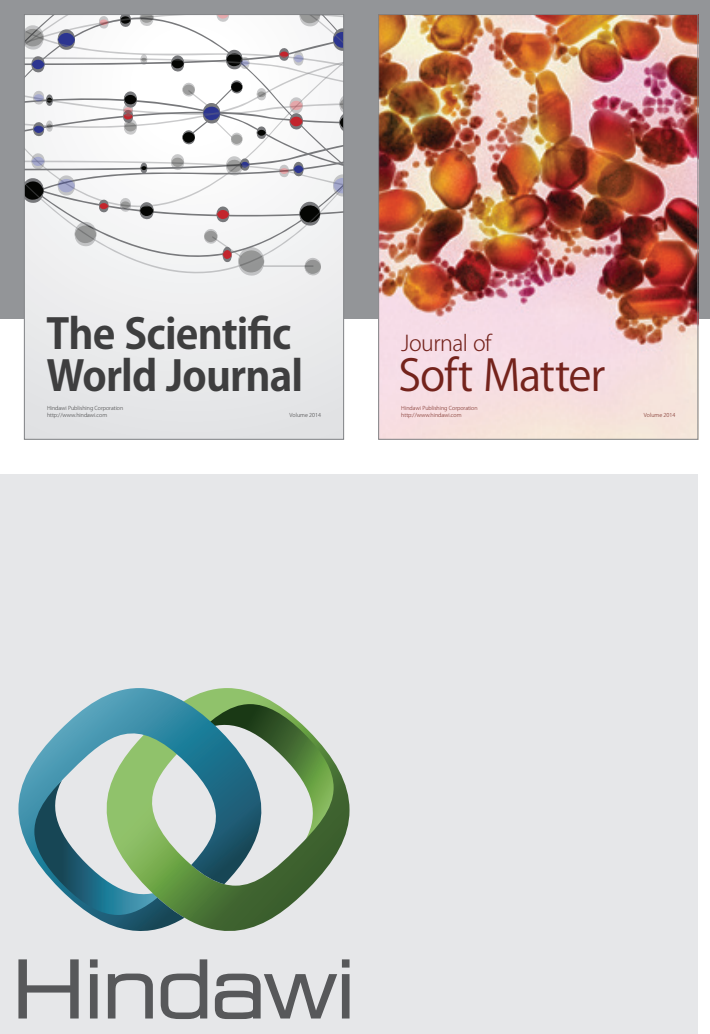

Submit your manuscripts at

http://www.hindawi.com
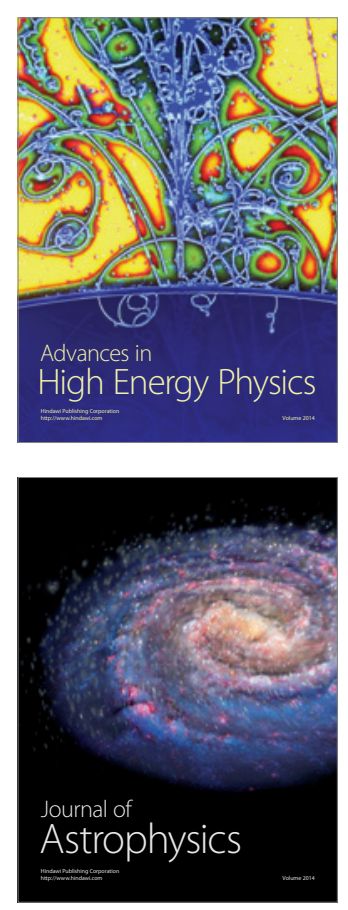
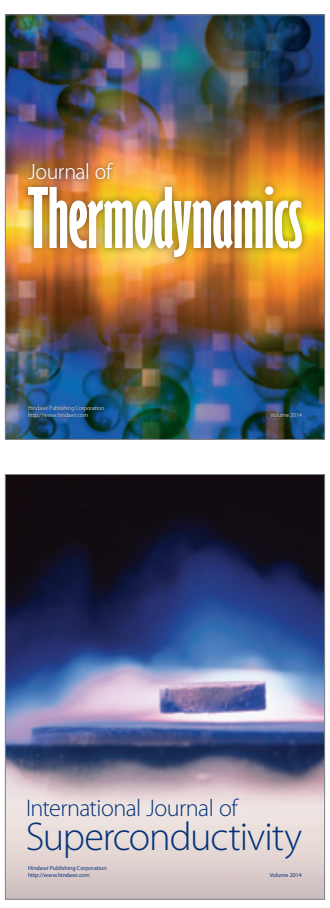
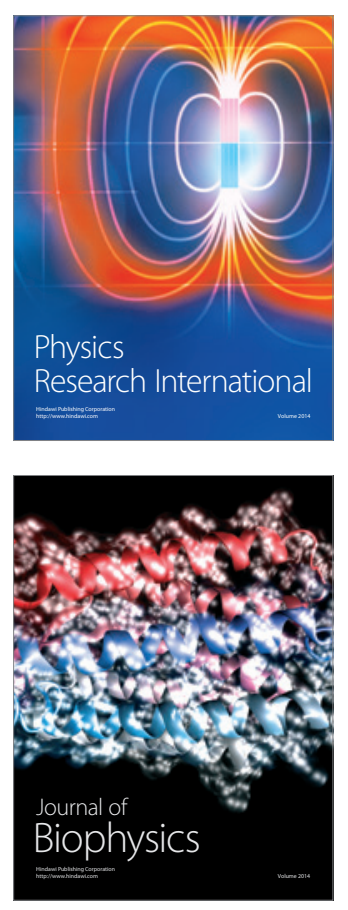
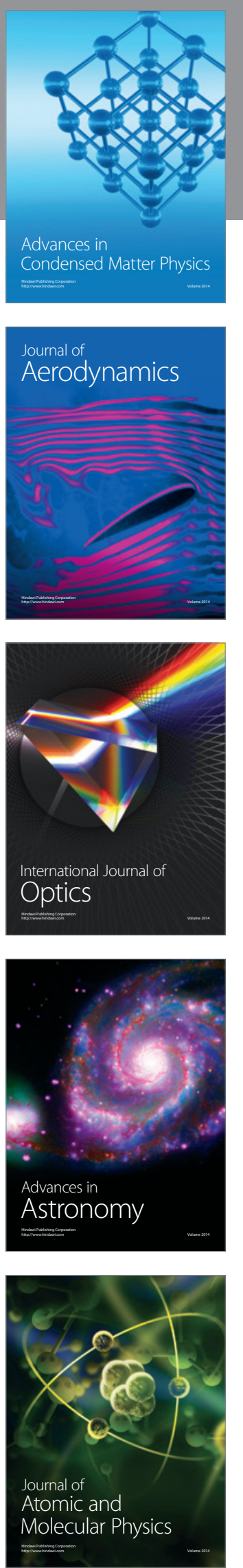